\title{
Classical and cubic Rashba effect in the presence of in-plane $4 f$ magnetism at the iridium silicide surface of the antiferromagnet $\mathrm{GdIr}_{2} \mathrm{Si}_{2}$
}

\author{
S. Schulz $\odot,{ }^{1, *}$ A. Yu. Vyazovskaya $\odot,{ }^{2,3}$ G. Poelchen $\odot,{ }^{1,4}$ A. Generalov,${ }^{5}$ M. Güttler, ${ }^{1}$ M. Mende,${ }^{1}$ S. Danzenbächer, ${ }^{1}$ \\ M. M. Otrokov ${ }^{6,7}$ T. Balasubramanian, ${ }^{5}$ C. Polley, ${ }^{5}$ E. V. Chulkov, ${ }^{8,3,9,6,2}$ C. Laubschat, ${ }^{1}$ M. Peters,${ }^{10}$ K. Kliemt $\odot,{ }^{10}$ \\ C. Krellner, ${ }^{10}$ D. Yu. Usachov $\odot,{ }^{3}$ and D. V. Vyalikh $\oplus^{9,7}$ \\ ${ }^{1}$ Institut für Festkörper- und Materialphysik, Technische Universität Dresden, 01069 Dresden, Germany \\ ${ }^{2}$ Tomsk State University, Lenina Avenue 36, 634050 Tomsk, Russia \\ ${ }^{3}$ St. Petersburg State University, 7/9 Universitetskaya nab., St. Petersburg, 199034, Russia \\ ${ }^{4}$ European Synchrotron Radiation Facility (ESRF), Avenue des Martyrs 71, 38043 Grenoble, France \\ ${ }^{5}$ Max IV Laboratory, Lund University, Box 118, 22100 Lund, Sweden \\ ${ }^{6}$ Centro de Física de Materiales (CFM-MPC), Centro Mixto CSIC-UPV/EHU, 20018 San Sebastián/Donostia, Spain \\ ${ }^{7}$ IKERBASQUE, Basque Foundation for Science, 48011 Bilbao, Spain \\ ${ }^{8}$ Departamento de Física de Materiales, Facultad de Ciencias Químicas, UPV/EHU, 20080 Donostia-San Sebastián, Basque Country, Spain \\ ${ }^{9}$ Donostia International Physics Center (DIPC), 20018 Donostia-San Sebastián, Basque Country, Spain \\ ${ }^{10}$ Kristall- und Materiallabor, Physikalisches Institut, Goethe-Universität Frankfurt, Max-von-Laue Straße 1, \\ 60438 Frankfurt am Main, Germany
}

(Received 31 August 2020; revised 3 December 2020; accepted 18 December 2020; published 15 January 2021)

\begin{abstract}
We present a combined experimental and theoretical study of the two-dimensional electron states at the iridium-silicide surface of the antiferromagnet $\mathrm{GdIr}_{2} \mathrm{Si}_{2}$ above and below the Néel temperature. Using angleresolved photoemission spectroscopy (ARPES) we find a significant spin-orbit splitting of the surface states in the paramagnetic phase. By means of ab initio density-functional-theory (DFT) calculations we establish that the surface electron states that reside in the projected band gap around the $\bar{M}$ point exhibit very different spin structures which are governed by the conventional and the cubic Rashba effect. The latter is reflected in a triple spin winding, i.e., the surface electron spin reveals three complete rotations upon moving once around the constant energy contours. Below the Néel temperature, our ARPES measurements show an intricate photoemission intensity picture characteristic of a complex magnetic domain structure. The orientation of the domains, however, can be clarified from a comparative analysis of the ARPES data and their DFT modeling. To characterize a single magnetic domain picture, we resort to the calculations and scrutinize the interplay of the Rashba spin-orbit coupling field with the in-plane exchange field, provided by the ferromagnetically ordered $4 f$ moments of the near-surface Gd layer.
\end{abstract}

DOI: 10.1103/PhysRevB.103.035123

\section{INTRODUCTION}

Spin-orbit coupling (SOC) in a noncentrosymmetric environment leads to the momentum-dependent splitting of otherwise spin-degenerate electron-energy bands that is known as Rashba SOC effect [1-3]. The spin degeneracy is lifted not only for the itinerant $s p d$-electron states [4], but the narrow bands originating from the highly localized $4 f$ states which are intrinsic for the heavy-fermion materials exhibit the fine spin splitting, too [5]. As beautiful playground for systematic studies of the emergence of the Rashba SOC effect and its properties serves the Si-terminated (001) surface of $\mathrm{RET}_{2} \mathrm{Si}_{2}$ intermetallic materials (RE - rare earth, $\mathrm{T}$ - transition metal), where the strength of the Rashba SOC can be tuned by choosing suitable transition metal atoms. It increases by exchanging $\mathrm{Co}$ for $\mathrm{Rh}$ atoms and further for Ir. Consequently, the Rashba effect will be weak for Co $3 d$ electrons, while it will be greatly enhanced for Ir $5 d$ orbitals. Further, combination of

*Corresponding author: susanne.schulz@tu-dresden.de the Rashba SOC effect with exchange magnetic [6] or Kondo interactions [5] allows for a gentle tuning of the spin-polarized carriers' properties such as their group velocity and spin structure [5-8].

In the recent past, we have performed a systematic study of the surface electronic structure of the aforementioned $\mathrm{RET}_{2} \mathrm{Si}_{2}$ systems by means of angle- and spin-resolved photoemission spectroscopy (ARPES and SARPES) [6,7,9-14]. Complementary theoretical studies in the frame of density functional theory (DFT) and effective $\mathbf{k} \cdot \mathbf{p}$ models have been proven powerful tools for gaining a deeper understanding of the formation of the surface states and their properties $[7,15,16]$. We believe that the remarkable agreement between experimental and theoretical studies obtained for $\mathrm{RET}_{2} \mathrm{Si}_{2}$ systems creates a sound basis for further investigations and the search for materials where similar or more unusual magnetic properties at the surface can be established theoretically first by means of DFT computations.

At the surface of $\mathrm{RET}_{2} \mathrm{Si}_{2}$ materials, the two-dimensional electron states form in the topmost Si-T-Si-RE block with 
a large contribution of transition metal $d$ states, which appear to be the driving force for the Rashba SOC. In that regard, an increase of the surface states' Rashba splitting is achieved by replacing the $4 d$ element $\mathrm{Rh}(Z=45)$ by isoelectronic, $5 d \operatorname{Ir}(Z=77)$. For both $\mathrm{Rh}$ and $\mathrm{Ir}$ compounds the splitting of the surface electron bands comes along with a characteristic spin polarization. In a textbook example of a classical Rashba system, i.e. the nearly-free two-dimensional electron gas, spin and momentum $\mathbf{k}$ are locked orthogonal to each other and spin performs one complete rotation by $2 \pi$ around the circular constant-energy contours (CECs) without changing its chirality [1]. Thus, for a classical Rashba system, we speak of a single winding of the electron spin around the CECs. A less common realization of the Rashba effect was recently discovered in the $\mathrm{RET}_{2} \mathrm{Si}_{2}$ family of materials [17]. Within a combined ARPES and DFT study performed on the iridium-silicide surface of the mixed-valent material $\mathrm{EuIr}_{2} \mathrm{Si}_{2}$ [6], for one of the surface states residing in a large projected gap around the $\overline{\mathrm{M}}$ point an unusual triple winding of the electron spin along the CECs was reported. The peculiar property of $\mathrm{EuIr}_{2} \mathrm{Si}_{2}$ is that being nonmagnetic in the bulk, it reveals strong and tunable magnetism at the surface where both SOC and exchange magnetism are involved. Moreover, its Si- and Eu-terminated surfaces and the respective subsurface areas reveal properties and temperature scales that are quite different from each other and from the bulk of the crystal [18]. Shortly afterwards, the aforementioned and computationally determined triple winding spin structure has been experimentally confirmed by an SARPES study of the similar surface state in the antiferromagnet $\mathrm{TbRh}_{2} \mathrm{Si}_{2}$ [7].

The triple winding spin structure consists in three complete rotations of the electron spin by $2 \pi$ upon moving once around the CECs. To gain deeper insight into the origin of the triple winding and the underlying difference to a single rotation of the spin a $\mathbf{k} \cdot \mathbf{p}$ model was introduced [7]. The respective $(2 \times 2)$ Hamiltonian includes two terms which account for the Rashba-like SOC: a term linear in $k, H_{\mathrm{R}}^{(1)} \propto \sigma \cdot \mathcal{B}_{\mathrm{R}}^{(1)}$, with the effective magnetic field $\mathcal{B}_{\mathrm{R}}^{(1)}=k\left(\sin \varphi_{\mathbf{k}},-\cos \varphi_{\mathbf{k}}, 0\right)$, and a term with a $k^{3}$ dependence, $H_{\mathrm{R}}^{(-3)} \propto \boldsymbol{\sigma} \cdot \mathcal{B}_{\mathrm{R}}^{(-3)}$, with the effective magnetic field $\mathcal{B}_{\mathrm{R}}^{(-3)}=k^{3}\left(\sin 3 \varphi_{\mathbf{k}}, \cos 3 \varphi_{\mathbf{k}}, 0\right)$. Here, $\boldsymbol{\sigma}$ is the vector of Pauli matrices and $\varphi_{\mathbf{k}}$ describes the polar angle of $\mathbf{k}$ with respect to the $\overline{\mathrm{M}}$ point of the surface Brillouin zone (SBZ). Value and sign of the superscript in $H_{\mathrm{R}}$ and $\mathcal{B}_{\mathrm{R}}$ refer to number and sense of rotation of the spin around the CECs (for a detailed description see below, Sec. III C). For the complete description of the $\mathbf{k} \cdot \mathbf{p}$ model we refer to Ref. [7]. The effective magnetic field aligns the spins parallel or antiparallel with the field direction. Thus the term with the linear dependence on $k$ results in a single winding of the spin, also referred to as linear Rashba effect, while the triple winding is a consequence of the $k^{3}$-dependent term and will therefore be referred to as cubic Rashba effect. Note that the surface states are subject to both the linear and the cubic Rashba effect simultaneously, but typically one of the two dominates. We also wish to note that an unusual spin structure, which is not described by the conventional Rashba model has been already detected for semiconducting and metallic two-dimensional electron systems $[19,20]$.
Further, for many members of the $\mathrm{RET}_{2} \mathrm{Si}_{2}$ family the 4f moments order magnetically at low temperatures. Most of these systems are antiferromagnets with ferromagnetically ordered rare-earth planes stacked antiferromagnetically along the $c$ axis [21-23]. The interplay between the emerging magnetic order of the $4 f$ moments in the fourth, subsurface layer and the itinerant surface state electrons leads to fascinating modifications of the Rashba-induced spin polarization and energy splittings which strongly depend on the actual orientation of the $4 f$ magnetic moments [24-27]. In $\mathrm{TbRh}_{2} \mathrm{Si}_{2}$, the $4 f$ moments point along the $c$ axis. The $c$ axis thus defines the direction in which the exchange field acts on the spins of the surface electrons, which, as a result, loose their in-plane locking and acquire a large $S_{z}$ component. The modified spin texture is accompanied by an enhancement of the energy splittings. Since the out-of-plane exchange field is perpendicular to the in-plane spin-orbit field, the triple winding of the inplane spin components is preserved in the magnetic phase of $\mathrm{TbRh}_{2} \mathrm{Si}_{2}$.

Even more interesting is the situation in which spin-orbit and exchange fields both act within the $a b$ plane as it is the case in $\mathrm{EuIr}_{2} \mathrm{Si}_{2}$ or $\mathrm{GdIr}_{2} \mathrm{Si}_{2}$. The in-plane magnetic order breaks the $C_{4 v}$ symmetry of the surface. ARPES studies of the $\mathrm{Si}$ surface in $\mathrm{EuIr}_{2} \mathrm{Si}_{2}$ revealed that this, in combination with SOC, leads to strong asymmetries in the band dispersions along those paths in the SBZ that run perpendicular to the magnetization direction, [100] [6]. The potential energy of a magnetic moment in a magnetic field $\mathbf{B}$ is $E=-\boldsymbol{\mu} \mathbf{B}$. If we identify $\boldsymbol{\mu}$ with the magnetic moment of the spin and $\mathbf{B}$ with the effective field describing the exchange interaction, we find that directly coupled states with a moment parallel to $\mathbf{B}$ are lowered and states with a moment antiparallel to $\mathbf{B}$ are raised in energy. For a more elaborate description we refer to Ref. [28]. Here, we would like to point out that in $\mathrm{RET}_{2} \mathrm{Si}_{2}$ systems bulk magnetism is not a necessary condition for magnetically active surface states. The surface state electrons are only sensitive to the subsurface rare earth layer (fourth atomic layer). As it was already mentioned, mixed-valent $\mathrm{EuIr}_{2} \mathrm{Si}_{2}$ has a nonmagnetic ground state because the valence of the $\mathrm{Eu}$ atoms fluctuates between the magnetic $\mathrm{Eu}^{2+}$ and nonmagnetic $\mathrm{Eu}^{3+}$ configurations $[29,30]$. Only in the subsurface layer Eu behaves divalently and its $4 f$ moments order magnetically below $48 \mathrm{~K}$, making the observation of the discussed effect possible [6].

In this paper, we focus on the new antiferromagnetic material $\mathrm{GdIr}_{2} \mathrm{Si}_{2}$ [21], where the spin properties of the surface electron states at its iridium-silicide surface are governed by both SOC and exchange magnetism of similar strength, that is in difference to $\mathrm{TbRh}_{2} \mathrm{Si}_{2}$ mentioned above [7]. Also, in the AFM phase of the $\mathrm{TbRh}_{2} \mathrm{Si}_{2}$ system, the $\mathrm{Tb} 4 f$ moments are oriented along the $c$ axis due to their coupling to the crystal-electric field. For $\mathrm{GdIr}_{2} \mathrm{Si}_{2}$, we will explore both the cubic and the classical Rashba effect and study how the spin structure of the surface states will be affected when the strong and robust in-plane magnetism from the $\mathrm{Gd} 4 f$ moments sets in. Nowadays, precise attention to the antiferromagnetic systems, and in particular, to their magnetically active surfaces, is motivated by the rapidly emerging subfield of spintronics that deals with antiferromagnets [31]. In regard of $\mathrm{GdIr}_{2} \mathrm{Si}_{2}$, we also wish to add that this material 
seems quite attractive for investigating magnetic domains and their properties at its iridium-silicide surface. For example: How do the domains appear and evolve with temperature? How does the surface couple to domains in the bulk? [32] What kind of domain walls are formed? [33,34] All these points could be a subject of further studies on $\mathrm{GdIr}_{2} \mathrm{Si}_{2}$. We believe that interesting fundamental physics linked to magnetic domains can be uncovered, and further application of nanospot techniques like nano-ARPES and spin-resolved photoemission electron microscopy (PEEM) could help to unravel them.

More precisely now, we explore here the spin properties of the two-dimensional electron states trapped at the $\mathrm{Si}-\mathrm{Ir}-\mathrm{Si}-\mathrm{Gd}$ surface block of the antiferromagnet $\mathrm{GdIr}_{2} \mathrm{Si}_{2}$ $\left(T_{\mathrm{N}}=86 \mathrm{~K}\right)$ for the paramagnetic $(\mathrm{PM})$ and antiferromagnetic (AFM) phases. For this purpose, we employ ARPES and DFT. The respective properties are governed: exclusively by the Rashba SOC for the PM phase, and by the joint action of Rashba and exchange fields for the AFM phase of $\mathrm{GdIr}_{2} \mathrm{Si}_{2}$. Like in many other $\mathrm{RET}_{2} \mathrm{Si}_{2}$ materials, the magnetic structure of $\mathrm{GdIr}_{2} \mathrm{Si}_{2}$ consists in ferromagnetic Gd layers in the $a b$ planes coupled antiferromagnetically to each other along the $c$ axis and separated by Si-Ir-Si trilayers [21]. Note, that since the orientation of the magnetization stemming from the ordered Gd $4 f$ magnetic moments within the surface Si-IrSi-Gd block remained unknown, we considered two cases in our DFT calculations. Namely, we have evaluated the spin structure of the surface electron states in the AFM phase when the staggered magnetization is directed along the [100] and [110] directions within the basal $a b$ plane. We considered these two limiting cases for the following reason: In light of our recently performed time-resolved experiments on the antiferromagnet $\mathrm{GdRh}_{2} \mathrm{Si}_{2}$ [32], the direction of the $\mathrm{Gd} 4 f$ moments reveals a temperature dependence which changes the easy magnetization axis. Such a "swimming" property of the magnetization as a function of temperature is not yet known for $\mathrm{GdIr}_{2} \mathrm{Si}_{2}$ but it cannot be excluded.

We wish to note that although the surface electronic structure for the PM phase of $\mathrm{GdIr}_{2} \mathrm{Si}_{2}$ can be quite nicely explored experimentally by means of conventional ARPES, its application to the AFM phase turns out to be quite challenging due to the appearance of multiple magnetic domains and steps at the surface. This leads to the observation of a complicated surface band structure in the ARPES patterns, which is difficult to reliably disentangle. Before, we experienced similar difficulties in the frame of our studies on $\operatorname{EuIr}_{2} \mathrm{Si}_{2}$. However, for $\mathrm{EuIr}_{2} \mathrm{Si}_{2}$ we were still able to obtain data from a single magnetic domain. This was not the case for $\mathrm{GdIr}_{2} \mathrm{Si}_{2}$ since the AFM order essentially complicates such a direct study. In the following, we will therefore discuss the AFM phase mainly in the frame of our theoretical results obtained from DFT calculations. In the end, thorough DFT modeling of different domain scenarios and comparison to our experimental results gives strong evidence that at $23 \mathrm{~K}$ the easy magnetization axis is along the [110] direction.

\section{COMPOUND INFORMATION AND METHODS}

$\mathrm{GdIr}_{2} \mathrm{Si}_{2}$ crystallizes in the tetragonal body-centered $\mathrm{ThCr}_{2} \mathrm{Si}_{2}$-type structure, characterized by space group
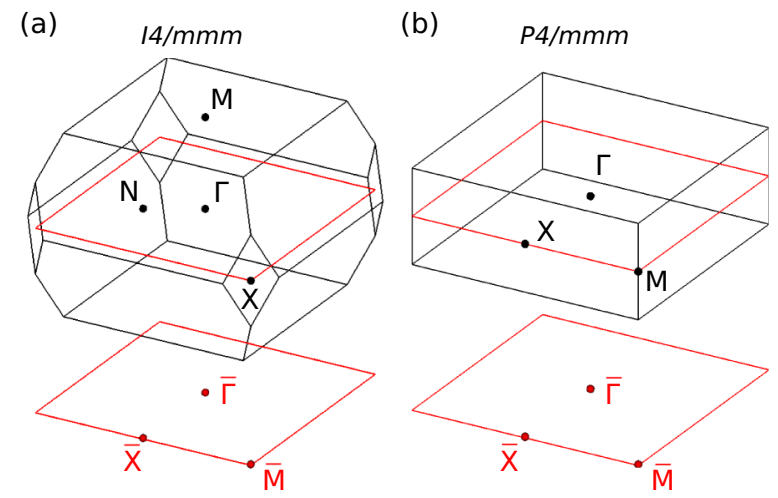

(c)

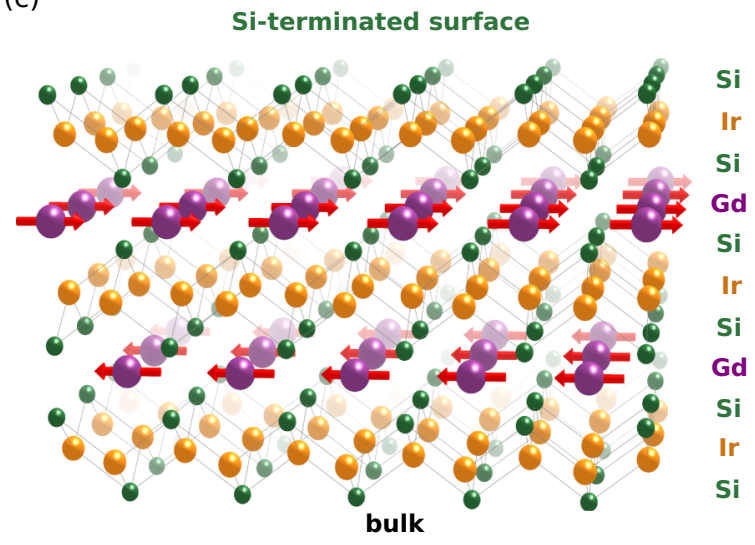

FIG. 1. Bulk (black) and surface (red) Brillouin zones for (a) $I 4 / \mathrm{mmm}$ and (b) $P 4 / \mathrm{mmm} \mathrm{GdIr}_{2} \mathrm{Si}_{2}$ unit cells. (c) Crystal structure showing the Si-terminated (001) surface of $\mathrm{GdIr}_{2} \mathrm{Si}_{2}$. Arrows indicate the AFM order of the Gd $4 f$ moments. For clarity, the moments are exemplary drawn collinear with the [100] direction.

$I 4 / \mathrm{mmm}$. The interlayer antiferromangetic order reduces the symmetry to $P 4 / \mathrm{mmm}$. Corresponding bulk and surface Brillouin zones (SBZ) are shown in Figs. 1(a) and 1(b). The $\mathrm{Gd}$ moments order ferromagnetically within the $a b$ plane while the actual in-plane orientation of the moments is not experimentally clarified yet. Consecutive Gd planes are well separated by $\mathrm{Si}-\mathrm{Ir}-\mathrm{Si}$ trilayer blocks and stacked antiferromagnetically along the $c$ axis. The crystal structure of the Si-terminated (001) surface of $\mathrm{GdIr}_{2} \mathrm{Si}_{2}$ is presented in Fig. 1(c).

For our ARPES experiments we cleaved $\mathrm{GdIr}_{2} \mathrm{Si}_{2}$ single crystalline samples [21] in situ under ultra-high-vacuum conditions. The ARPES experiments were performed at the Bloch beamline at the MAX IV laboratory [35]. The data for the PM phase were acquired at a temperature of about $120 \mathrm{~K}$, while the measurements for the AFM phase were performed at a temperature of about $23 \mathrm{~K}$. In both cases, we used linear horizontal light polarization and a photon energy of $h v=55 \mathrm{eV}$.

The electronic structure was calculated in the frame of DFT using the full-potential local-orbital minimum-basis code FPLO [36]. We applied the DFT $+U$ approach within the generalized gradient approximation (GGA) of Perdew, Burke, and Ernzerhof for the exchange-correlation functional, and $U=6.7 \mathrm{eV}, J=0.7 \mathrm{eV}$ (Slater parameters $F_{0}=6.7, F_{2}=$ $8.3, F_{4}=5.7, F_{6}=4.1$ ) for the Gd $4 f$ electrons. Starting 


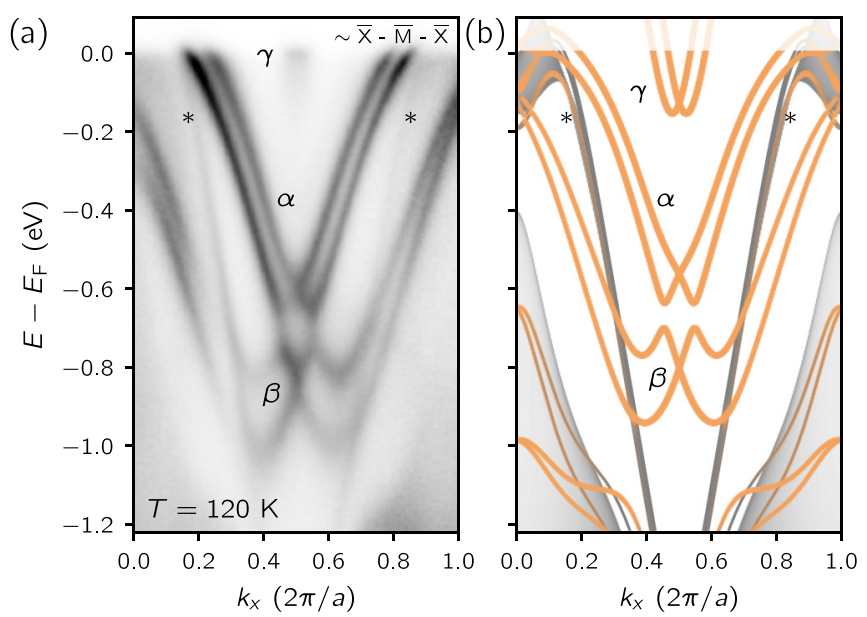

FIG. 2. Surface band structure along $\overline{\mathrm{X}}-\overline{\mathrm{M}}-\overline{\mathrm{X}}$ for the Siterminated (001) surface of $\mathrm{GdIr}_{2} \mathrm{Si}_{2}$. (a) The surface states $\alpha$, $\beta$ and $\gamma$ measured with ARPES $(h v=55 \mathrm{eV})$ in the PM phase $(T=120 \mathrm{~K})$ are nicely captured by the DFT calculation, shown in (b) for a slab of 16 atomic layers. There, surface or surface-resonant states are highlighted in orange, while the bulk bands projected onto the surface Brillouin zone are shown as a light gray shading. In the ARPES data shown in (a), each energy-distribution curve is normalized to the same total intensity. The asterisks $\left(^{*}\right)$ mark a surface resonant state at the edge of the band gap to which $\alpha, \beta$, and $\gamma$ are confined.

from the experimental values for the lattice parameters, we minimized the total energy with respect to the lattice constants $a$ and $c$ as well as the $z$ position of $\mathrm{Si}$ in a scalar-relativistic setting for the antiferromagnetic configuration. The obtained values for $a, c$ and $z_{\mathrm{Si}}$ deviate from the experimental ones by about $+1 \%$. In all further calculations the optimized lattice parameters were used. Fully relativistic calculations were performed for bulk and surface electronic structure. To model the surface we built a slab of 16 atomic layers with a Si- and a Gd-terminated surface, well separated by a vacuum of $22 \AA$. All layers of the slab were relaxed to account for relaxation of the surface layers. For the PM phase, we applied the open core approach with an unpolarized $4 f$ shell. That is, the $4 f$ electrons are removed from the valence basis and treated as core electrons with a fixed occupancy. The correctness and completeness of all results was validated by comparison to respective calculations using the projector augmented wave (PAW) method (VASP code) [37,38], where the basis set consists of plane waves.

\section{RESULTS AND DISCUSSION}

\section{A. Dispersion of the surface states in the paramagnetic phase}

We begin with introducing the surface states of Siterminated $\mathrm{GdIr}_{2} \mathrm{Si}_{2}(001)$. A comparison with spectra taken from a Gd-terminated surface can be found in Ref. [39] (Fig. S1). In Fig. 2(a), we show a respective ARPES map taken along $\overline{\mathrm{X}}-\overline{\mathrm{M}}-\overline{\mathrm{X}}$ at a temperature of $120 \mathrm{~K}$, that is notably above the Néel temperature $T_{\mathrm{N}}=86 \mathrm{~K}$. In Fig. 2(b), the corresponding DFT band structure is given. The surface states residing in the projected bulk band gap are labeled $\alpha, \beta$, and $\gamma$. They are clearly distinguishable in the ARPES map and well reproduced by the DFT calculation. Only for the $\gamma$ state there is some discrepancy in binding energy between experiment and theory. Namely, ARPES reveals that only the very bottom of the band is occupied while the larger, unoccupied part of the $\gamma$ band lies above the Fermi level and is thus not accessible to photoemission. In the calculation, the band is shifted by about $150 \mathrm{meV}$ towards higher binding energies as compared to the experiment. The origin of this discrepancy lies in the surface relaxation effect. The $\gamma$ band is mainly formed by states of the surface $\mathrm{Si}$ (whose contribution is about $83 \%$ ) and the adjacent Ir layer (14\%). Therefore its energy position is extremely sensitive to the interlayer spacing between the two atomic layers. A slight shift of the topmost layer comes along with an energy shift of the $\gamma$ band. Apparently, DFT overestimates the interlayer spacing between the surface $\mathrm{Si}$ and the adjacent Ir layer causing the $\gamma$ band to appear at higher binding energies. For a detailed discussion of the relaxation effect we refer to Ref. [39] (Fig. S2 and related discussion).

The dispersion and energy positions of the $\alpha$ and $\beta$ states are nicely captured by the calculation. In the $\overline{\mathrm{M}}$ point, the $\alpha$ state is built by $\propto 50 \%$ of Si $3 p$, by $\propto 34 \%$ of Ir $5 d$ and by $\propto 7 \%$ of Gd $5 p$ and $\mathrm{Gd} 5 d$ derived orbitals. For the $\beta$ state, we find a similar orbital composition. The Rashba-like SOC manifests itself in a large and symmetric spin splitting of the $\alpha, \beta$ and $\gamma$ bands, that otherwise would be doubly degenerate (see Ref. [39], Fig. S4(a)).

\section{B. Dispersion of the surface states in the magnetic phase}

To clarify the changes of the surface states when going from the PM to the AFM phase, we compare in Fig. 3 ARPES spectra measured above $T_{\mathrm{N}}$ at $120 \mathrm{~K}$, Figs. 3(a) and 3(b), with spectra taken below $T_{\mathrm{N}}$ at $23 \mathrm{~K}$, Figs. 3(c) and 3(d). For this purpose, we show the high-symmetry cuts along $\overline{\mathrm{X}}-\overline{\mathrm{M}}-\overline{\mathrm{X}}$ in Figs. 3(a) and 3(c) for both phases "as measured", normalized (each energy-distribution curve was normalized to the same total intensity) and after curvature treatment [40]. Comparing the data for the AFM phase shown in Fig. 3(c) to the PM data in Fig. 3(a), we clearly see two more bands appearing for the $\alpha$ state, and in the vicinity of $\overline{\mathrm{M}}$, one additional band for $\beta$. This is best seen in the curvature plot and a clear indication for the presence of different magnetic domains or terraces at the surface, which are characterized by a different in-plane orientation of the magnetic moments in the subsurface Gd layer. When surface regions with different magnetization directions are probed simultaneously, the measured photoemission intensity map is a superposition of the bands associated with each single direction of magnetization. Since the band dispersion is highly nonsymmetric in case of an in-plane magnetization in the presence of a strong Rashba field (see the extensive discussion below), "additional" bands appear in the ARPES map. This is also well seen by comparing the Fermi surface maps shown in Figs. 3(b) and 3(d). Here, the star-shaped features are the CECs of the $\alpha$ band. In the Fermi map taken in the PM phase shown in Fig. 3(b) the inner and outer contour of the $\alpha$ state reflect the fourfold rotational symmetry of the crystal. In the AFM phase the $C_{4 v}$ symmetry of the surface is broken. In addition, we observe additional contour lines for $\alpha$ in Fig. 3(d). The superimposed bands are most clearly manifested near the corners of the four-pointed 

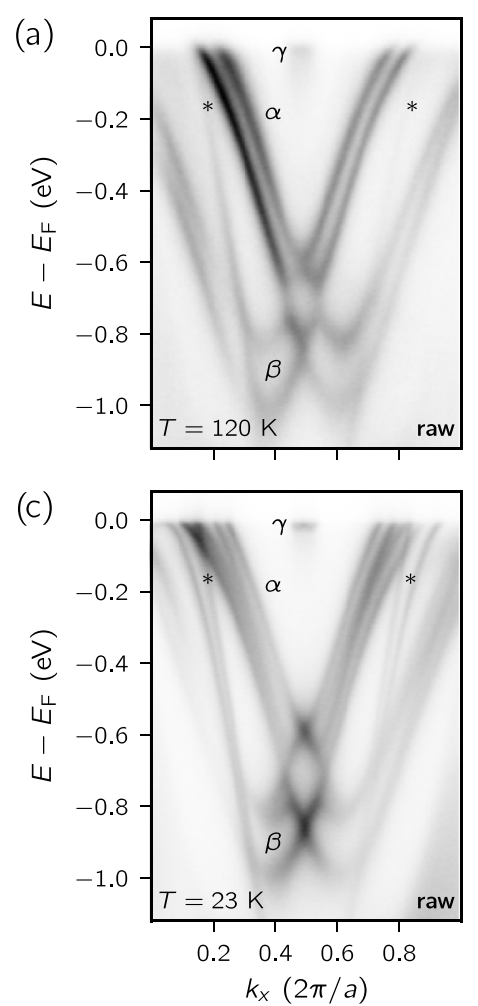
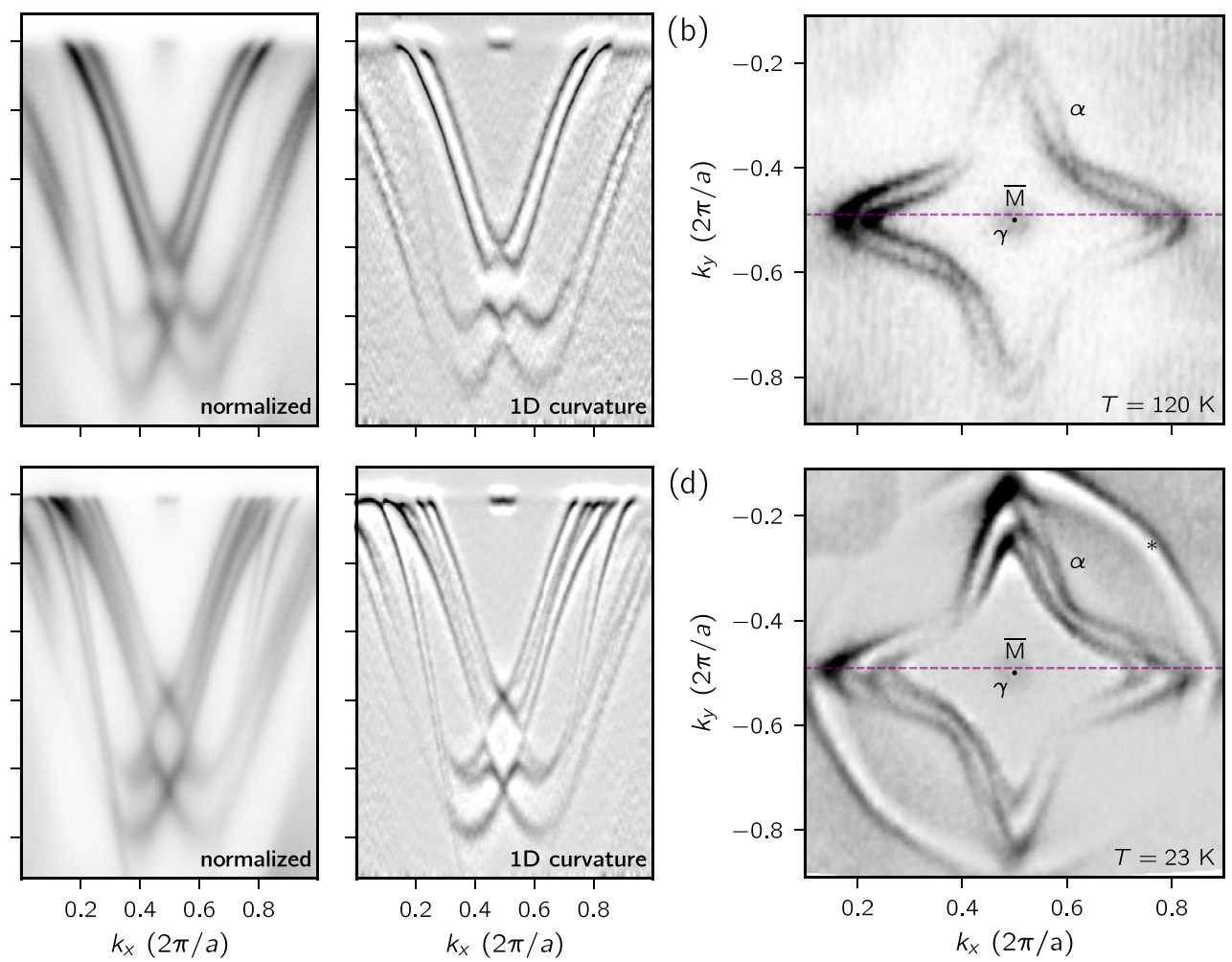

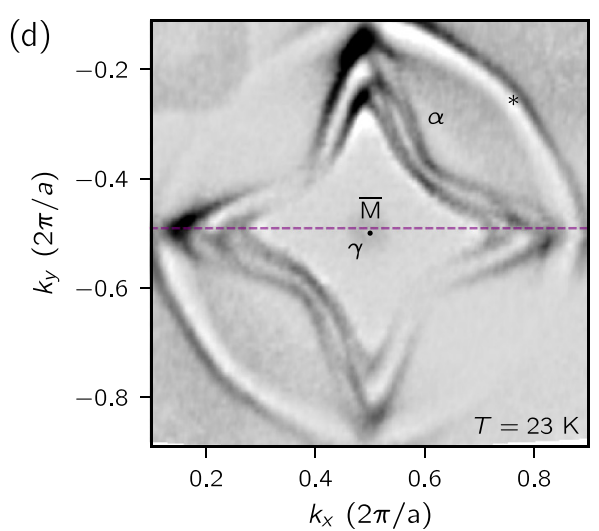

FIG. 3. ARPES view on the surface states $\alpha, \beta, \gamma$ and the surface resonant state $(*)$ along $\overline{\mathrm{X}}-\overline{\mathrm{M}}-\overline{\mathrm{X}}$ in $(\mathrm{a})$ the PM phase $(T=120 \mathrm{~K})$ and (c) the AFM phase $(T=23 \mathrm{~K})$. For comparison, the data are shown "as measured", after normalization of each energy-distribution curve to the total intensity (normalized) and after curvature treatment along the energy axis [40] using the same curvature parameters for both the PM and AFM phases. In (b) and (d), the ARPES-derived Fermi surface is shown in the PM and AFM phases, respectively. The star-shaped contours are formed by the $\alpha$, the weak intensity around the $\overline{\mathrm{M}}$ point originates from the $\gamma$ state. Purple-dashed lines indicate the $k$ paths that correspond to the cuts shown in (a) and (c).

star: around each corner one can observe four lines, i.e. two from the inner and two from the outer CEC. Note that in close vicinity to the $\overline{\mathrm{M}}$ point, also the Fermi contour of the $\gamma$ band is visible. We would like to add that the surface resonant state marked by asterisks (*) in Figs. 2 and 3 is apparently spin split, too. This effect has been seen and discussed before for $\mathrm{GdRh}_{2} \mathrm{Si}_{2}$ [12] and $\mathrm{HoRh}_{2} \mathrm{Si}_{2}$ [14] and is beyond the scope of this story.

The surface state electrons are sensitive to the magnetically ordered $4 f$ moments in the subsurface Gd layer (fourth atomic layer). This Gd layer is coupled via the Ruderman-KittelKasuya-Yosida (RKKY) interaction to the Gd layers in the bulk. If magnetic domains form in the bulk of the crystal, they will be inherited to the subsurface Gd layer and thus, affect the magnetic properties of the electrons in the surface. Moreover, after cleaving, the surface is not flat but consists of many terraces which are separated by steps of few atomic layers. Even in the case of a single magnetic domain in the bulk, the sole existence of these steps will result in the observation of antiparallel domains in the electronic structure of the surface, provided that the spot size of the probing photon beam is larger than a terrace. Since terraces at the surface cause the same macroscopic effect like magnetic domains, in the following the term domain will also be used in context of terraces. Hence, in the simplest case of a single magnetic domain in the bulk, this would result in the superposition of
ARPES patterns of two antiparallel domains, which is due to the AFM stacking of the Gd layers, and will be explored in comparison with DFT calculations in Sec. III D.

To directly measure a single magnetic domain, one could potentially try a more specialized experimental technique like nano-ARPES, which, on the other hand comes along with other experiment-specific difficulties. The presence of different magnetic domains at the $\mathrm{Si}$ surface was observed previously for $\mathrm{EuIr}_{2} \mathrm{Si}_{2}$ [6]. For the latter, we managed to separately acquire ARPES data from both a single magnetic domain and two oppositely magnetized domains. This was possible because $\mathrm{EuIr}_{2} \mathrm{Si}_{2}$ is not magnetic in the bulk but the Eu moments in the fourth atomic layer order magnetically such that exchange interaction with the surface state electrons can emerge. Since only the Eu atoms in the subsurface are magnetically active they are magnetically decoupled from the bulk, which reduces the possibilities and, hence, the likelihood for domain formation. In principle, one may even consider to reduce the number of magnetic domains by magnetizing the sample surface by applying an external magnetic field. In $\mathrm{GdIr}_{2} \mathrm{Si}_{2}$ we did not succeed in acquiring data from a single magnetic domain. In comparison to magnetically active divalent $\mathrm{Eu}, \mathrm{Gd}$ is trivalent with an additional $5 d$ electron which allows for a strong antiferromagnetic interlayer coupling via the RKKY interaction. The three-dimensional coupling makes it difficult to sufficiently increase domain sizes by 
external magnetic fields. However, experiments on $\mathrm{GdRh}_{2} \mathrm{Si}_{2}$ showed that in the temperature range between 60 and $90 \mathrm{~K}$ the magnetic moments in the bulk and at the surface couple rather weakly, such that bulk and surface magnetism can be considered independently [12]. If additional experiments can reveal a similar behavior in $\mathrm{GdIr}_{2} \mathrm{Si}_{2}$, one possibility for the observation of a single domain might be to magnetize and measure the sample at such an intermediate temperature. Note, that in the antiferromagnetic sister compound $\mathrm{EuRh}_{2} \mathrm{Si}_{2}$, where $\mathrm{Eu}$ is divalent, the ordering temperature of the surface is even above the ordering temperature of the bulk [11,14].

\section{Spin structure}

Since data from a single magnetic domain are experimentally not available for $\mathrm{GdIr}_{2} \mathrm{Si}_{2}$, we will employ DFT for the further exploration of its surface electronic structure, and particularly pay attention to how the spin texture and band dispersion evolve under the joint action of spin-orbit and exchange interaction on the surface electrons. DFT has proven to give an accurate description of the surface and bulk electronic structure of $\mathrm{RET}_{2} \mathrm{Si}_{2}$ compounds, and in particular also of $\mathrm{GdIr}_{2} \mathrm{Si}_{2}$ as can be seen in Fig. 2. Since the precise direction of the in-plane staggered magnetization easy axis is not known for $\mathrm{GdIr}_{2} \mathrm{Si}_{2}$, we will consider the cases of the easy axis pointing along [100] and [110]. In the following, we start our discussion from the spin structure in the PM phase.

\section{Spin structure in the PM phase}

In Fig. 4, we give an overview on the spin structure of the $\alpha$ and $\gamma$ surface states in the PM phase. A statement about the $\beta$ state will be given below. The upper panel of Fig. 4 shows each band along the $\overline{\mathrm{X}}-\overline{\mathrm{M}}-\overline{\mathrm{X}}$ direction, which is parallel to $k_{x}$ and highlighted in terms of its spin expectation value $S_{y}$. Taking a closer look at the spin structure of the $\alpha$ state one can notice that the spin polarization is close to zero in the vicinity of the $\overline{\mathrm{M}}$ point. Moreover, for a given $\mathbf{k}, S_{y}$ has the same sign on the upper and lower bands. The vanishing spin polarization as well as the identical sign of $S_{y}$ close to the $\overline{\mathrm{M}}$ point appear due to a hidden spin polarization [41]. The origin of this effect lies in the existence of two Ir sublattices in the same atomic plane (for a visualization of the two Ir sites see Ref. [39], Fig. S3), each of which is associated with a particular spin polarization [16]. Due to the symmetry of the crystal both contributions add up to the total spin polarization shown in Fig. 4. Since close to $\bar{M}$ the spin components have similar absolute values but opposite signs for each site, the two contributions almost cancel out [16]. Upon moving away from $\bar{M}$ the spin polarization increases and the typical Rashba pattern establishes. In the following, we restrict our discussion to $k$, which are sufficiently far from $\bar{M}$ and do not discuss the hidden polarization phenomenon.

Looking at the band dispersion, at first glance we recognize the characteristic spin pattern of a classical Rashba system, which can be phenomenologically described by two parabolas of opposite spin polarization whose vertices are shifted against each other by the same wave vector $\Delta k$. Indeed, the dispersion of $\gamma$ closely resembles such a picture, while the dispersion of $\alpha$ deviates more strongly from the parabolic
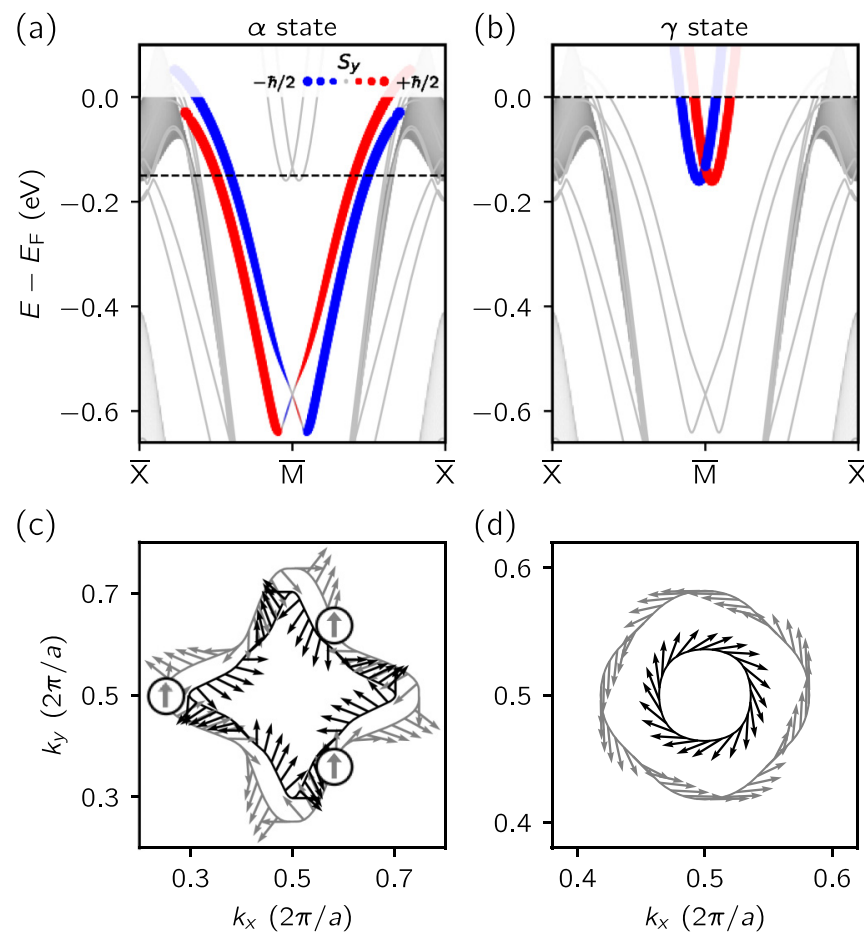

FIG. 4. Calculated spin structure of the surface states in the PM phase along $\overline{\mathrm{X}}-\overline{\mathrm{M}}-\overline{\mathrm{X}}$ parallel $k_{x}$. [(a) and (c)] $\alpha$ and [(b) and (d)] $\gamma$ states. In (a) and (b), slab and projected bulk bands are plotted as gray lines and areas, respectively. The surface state bands of $\alpha$ and $\gamma$ are highlighted by the spin expectation value $S_{y}$ in red $\left(S_{y}>0\right)$ and blue $\left(S_{y}<0\right)$. In (c) and (d), the spin structure along the CECs is shown. The energy of the CECs is marked by a black dashed line in the $E(k)$ plots above. Small encircled arrows indicate the triple winding of the spin on the CECs of $\alpha$.

law. To illustrate the key difference between the $\alpha$ and $\gamma$ states, we show in Figs. 4(c) and 4(d) the spin structures for their CECs. The energy of each CEC is marked by a black dashed line in the corresponding upper panel. For the $\gamma$ state, we observe that the spin rotates differently when compared to $\alpha$. For a consistent description of the rotation of the spin along a CEC, we introduce the following definitions. The winding number $n \in \mathbb{Z}$ is defined in such a way that $2 \pi n$ describes the angle of rotation of the spin when performing a closed loop along the contour. Since for a closed contour the spin at the start and end has to coincide, the winding number $n$ has to be an integer and therefore expresses the number of full rotations of the spin. On the other hand, the sign of $n$ represents the sense of rotation of the spin. When moving along the CEC in a counterclockwise direction, the spin can turn counterclockwise or clockwise. Hence, if the spin rotates in the same direction as the movement direction along the CEC, we define $n$ to be positive, while an opposite rotation of the spin to the movement along the CEC has a negative sign.

However, for the surface of $\mathrm{GdIr}_{2} \mathrm{Si}_{2}$, some winding numbers $n$ are forbidden by the underlying $C_{4 v}$ symmetry. The winding of the spin $\mathbf{S}(\varphi)$ along the CEC has to follow this four-fold symmetry of the crystal, meaning that the spin $\mathbf{S}\left(\varphi+\frac{\pi}{2}\right)=R_{z}\left(\frac{\pi}{2}\right) \mathbf{S}(\varphi)$ with $R_{z}$ being the rotation matrix 
about the rotation axis $z$. In other words, the spin needs to rotate by $2 \pi \eta+\frac{\pi}{2}$ with $\eta \in \mathbb{Z}$ when moving along the CEC by an angle of $\frac{\pi}{2}$. This way, the full rotation around the closed contour $2 \pi n=4\left(2 \pi \eta+\frac{\pi}{2}\right)$ leads to the allowed winding numbers $n=4 \eta+1$.

The simplest allowed winding of the spin is thus $n=1$ with $\eta=0$, meaning that the spin performs one full rotation when moving along the closed CEC. Here, the spin rotates in the same direction to the movement along the CEC. We will call this rotation henceforth single winding. The next lowest number of rotations is $n=-3$ for $\eta=-1$. In this case, the spin rotates oppositely to the direction taken along the closed CEC performing three full rotations. This rotation of the spin is therefore called triple winding. In principle, higher orders of rotation are possible with alternating sense of rotation $(n=5,-7,9 \ldots)$.

Based on this definition, we can now evaluate the CECs in Fig. 4(c) and Fig. 4(d) for $\alpha$ and $\gamma$, respectively. Starting with the inner contour of $\gamma$ shown in black, we can see that the spin rotates clockwise when moving along the contour in the clockwise direction and performs one full rotation of $2 \pi$ along the closed contour. The same applies to the outer contour of $\gamma$ shown in gray, hence we find the winding number to be $n_{\gamma}=1$ for inner and outer contour. For the more complex looking CECs of $\alpha$, we perform the analysis in the same way. Let us start at the uppermost point of the outer contour $\left(k_{x}=0.5 \frac{2 \pi}{a}\right.$, $k_{y} \approx 0.73 \frac{2 \pi}{a}$ ) with the spin pointing to the right along the $k_{x}$ direction. Moving now a small section along the contour in the clockwise direction, the spin starts to rotate counterclockwise pointing more and more to the top of the figure along the $k_{y}$ direction. Thus, the winding number has to be negative. Following the spin along the closed contour, it performs three full rotations, highlighted by the three encircled arrow marks of the spin pointing along the $k_{y}$ direction, which are related by a full rotation of the spin by $2 \pi$. The inner contour behaves in the same way, resulting in a winding number of $n=-3$ for $\alpha$.

To describe these spin-orbit induced spin structures, an effective Rashba-like Hamiltonian $H_{\mathrm{R}} \propto \mathcal{B}_{\mathrm{R}} \boldsymbol{\sigma}$ with $\mathcal{B}_{\mathrm{R}}$ as effective spin-orbit field can be applied. For a general description, we can expand $\boldsymbol{\mathcal { B }}_{\mathrm{R}}$ in terms of rotations $n_{\mathrm{R}}$ with $\mathcal{B}_{\mathrm{R}}^{\left(n_{\mathrm{R}}\right)}=\operatorname{sgn}\left(n_{\mathrm{R}}\right) k^{\left|n_{\mathrm{R}}\right|}\left(\sin n_{\mathrm{R}} \varphi_{\mathbf{k}},-\cos n_{\mathrm{R}} \varphi_{\mathbf{k}}, 0\right)$. Here, we clearly see that the different signs of $n_{\mathrm{R}}$ are connected by $\mathcal{B}_{\mathrm{R}}^{\left(n_{\mathrm{R}}\right)}=(1,-1,0) \mathcal{B}_{\mathrm{R}}^{\left(-n_{\mathrm{R}}\right)}$ expressing the different senses of rotation. Note that the sgn function is used for a consistent sign convention with Ref. [7] of the single and cubic Rashba term discussed in the following.

Since $\mathcal{B}_{\mathrm{R}}$ has to follow the crystal symmetry too, $n_{\mathrm{R}}=4 \eta+1 \quad(\eta \in \mathbb{Z})$. Hence, the lowest order term with $n_{\mathrm{R}}=1$ results in $\mathcal{B}_{\mathrm{R}}^{(1)}=k\left(\sin \varphi_{\mathbf{k}},-\cos \varphi_{\mathbf{k}}, 0\right)$, which corresponds to the classical linear Rashba term. The resulting spin structure of the linear Rashba term is a single winding of the spin making this the dominating term for the $\gamma$ band. The next higher order is $n_{\mathrm{R}}=-3$ with $\mathcal{B}_{\mathrm{R}}^{(-3)}=-k^{3}\left(\sin -3 \varphi_{\mathbf{k}},-\cos -3 \varphi_{\mathbf{k}}, 0\right)=k^{3}\left(\sin 3 \varphi_{\mathbf{k}}, \cos 3 \varphi_{\mathbf{k}}, 0\right)$ resulting in a triple winding of the spin. Hence, this is the dominant term to describe the spin structure of the $\alpha$ band. Due to its $k^{3}$ dependence, it is called the cubic Rashba term and thus the resulting effect for the triple winding being called the cubic Rashba effect. Higher orders can be used to describe more complex spin rotations [42].

That the leading contribution to $\mathcal{B}_{\mathrm{R}}$ differs strongly between the $\alpha$ and $\gamma$ states can be understood as a direct consequence of their different orbital composition. Indeed, applying the spin-orbit interaction as a small perturbation to the local-orbital eigenfunctions of the scalar-relativistic DFT calculation, we can reproduce the above spin structure as shown in Ref. [39] (Figs. S4-S6 and related discussion). We further observe that the orbital composition of the $\beta$ state leads to a spin structure dominated by the linear Rashba term, but still deviates significantly from the spin structure of the $\gamma$ state due to an additional contribution from the cubic Rashba term. The individual contributions were discussed quantitatively for $\mathrm{TbRh}_{2} \mathrm{Si}_{2}$ [7]. Therefore we will omit the discussion of the $\beta$ state here.

Lastly, we would like to note that, while it is quite common to drop the sign of expansion terms $n_{\mathrm{R}}[7,42]$ and use $\mathcal{B}_{\mathrm{R}}^{\left(n_{\mathrm{R}}\right)}$ interchangeably for $\mathcal{B}_{\mathrm{R}}^{\left(n_{\mathrm{R}}\right)}$ and $\mathcal{B}_{\mathrm{R}}^{\left(-n_{\mathrm{R}}\right)}$, our definition presented here removes this ambiguity. The same problem has to be kept in mind when using the names linear and cubic Rashba term as they can be ascribed to $\mathcal{B}_{\mathrm{R}}^{(1)}$ or $\mathcal{B}_{\mathrm{R}}^{(-1)}$ and $\mathcal{B}_{\mathrm{R}}^{(3)}$ or $\mathcal{B}_{\mathrm{R}}^{(-3)}$, respectively.

\section{Cubic Rashba effect and in-plane exchange field ( $\alpha$ state)}

For a detailed analysis of the dispersion and the spin structure of the $\alpha$ and $\gamma$ surface states, we consider the spinresolved bands and CECs calculated for the PM and AFM phases. Keep in mind that $\mathrm{GdIr}_{2} \mathrm{Si}_{2}$ is a layered antiferromagnet, which means that the sign of the magnetization in the ferromagnetically ordered Gd layers alternates in consecutive Gd layers. Since the electrons in the surface Si-Ir-Si-Gd block are only magnetically sensitive to the fourth atomic layer $(\mathrm{Gd})$, the precise direction of magnetization in the latter is essential for our analysis of the spin and electronic structure of the states $\alpha$ and $\gamma$. We consider the two cases in which the magnetization in the subsurface Gd layer is along [100] or [110].

Respective plots are shown in Fig. 5. CECs are presented for $E=-0.15 \mathrm{eV}$ and $E=0 \mathrm{eV}$ for $\alpha$ and $\gamma$, respectively. While in Fig. 4 the spin textures of the CECs are presented by arrows, in Fig. 5, we use a complementary representation, in which the individual spin components $S_{x}$ and $S_{y}$ are shown. On the right of the CECs, we show the dispersion and spin structure of the surface states for two pairs of the $\bar{X}-\bar{M}-\bar{X}$ and $\bar{\Gamma}-\overline{\mathrm{M}}-\bar{\Gamma}$ directions indicated in Figs. 5(a) and 5(d), thereby going from the left to the right (i.e., from the subpanel " 1 " to subpanel "4") Note that the high-symmetry directions shown in successive subpanels are related by a counterclockwise rotation by $\pi / 4$ around $\bar{M}$.

As can be seen in Fig. 5(a) in the PM phase $\alpha$ demonstrates a symmetrical dispersion with respect to the $\overline{\mathrm{M}}$ point for both pairs of $\overline{\mathrm{X}}-\overline{\mathrm{M}}-\overline{\mathrm{X}}$ and $\bar{\Gamma}-\overline{\mathrm{M}}-\bar{\Gamma}$ directions. Namely, due to fourfold rotational symmetry the band dispersion is the same for mutually orthogonal $k$ paths. The spin polarization behavior is consistent with the cubic Rashba effect. For the $\overline{\mathrm{X}}-\overline{\mathrm{M}}-\overline{\mathrm{X}} \| k_{x}$ direction, the spins are polarized along $\hat{y}$, while for the $\overline{\mathrm{X}}-\overline{\mathrm{M}}-\overline{\mathrm{X}}$ $\| k_{y}$ path, in contrary, along $\hat{x}$. For the $\bar{\Gamma}-\overline{\mathrm{M}}-\bar{\Gamma}$ directions, both $S_{x}$ and $S_{y}$ spin components are equally presented. Thus the line 

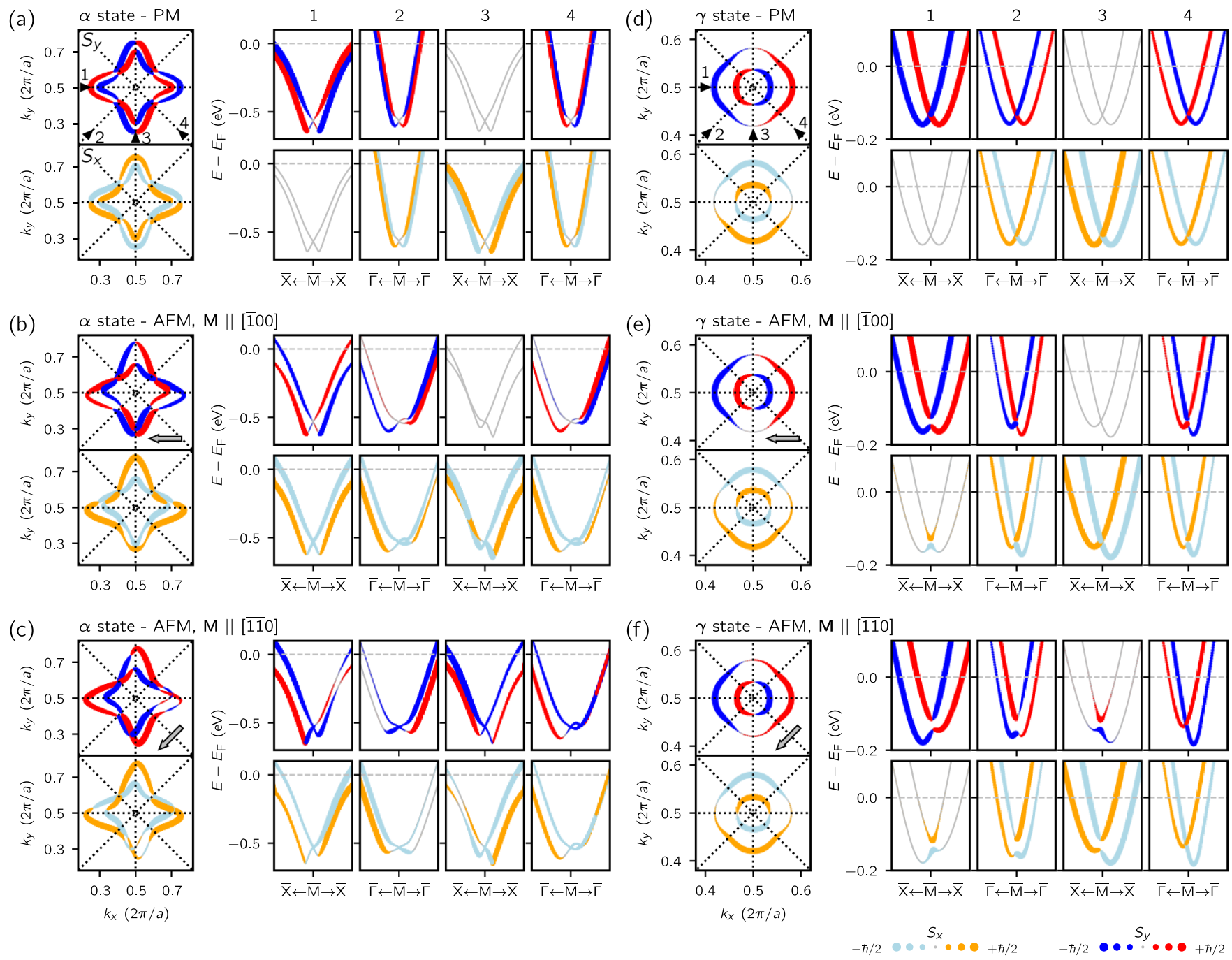

FIG. 5. Spin-resolved CECs and band structure of the $\alpha$ and $\gamma$ states. For the $\alpha$ state CECs and band structure are shown in (a) for the PM phase, and in (b) and (c) for the AFM phases with magnetization direction along [100] and [110], respectively. In (d)-(f), respective information is given for the $\gamma$ state. CECs are presented for $E=-0.15 \mathrm{eV}$ and $E=0 \mathrm{eV}$ for $\alpha$ and $\gamma$, respectively. The surface state bands are highlighted by the spin expectation value $S_{x}$ in orange $\left(S_{x}>0\right)$ and light blue $\left(S_{x}<0\right)$ and $S_{y}$ in red $\left(S_{y}>0\right)$ and blue $\left(S_{y}<0\right)$. The line width scales with the absolute value of the spin expectation value. The chosen high-symmetry directions in the SBZ, which are numbered from 1 to 4 , are indicated by black dotted lines in the plots of the CECs. In (a) and (d), arrows mark the direction along which the $k$ path is shown. Large gray arrows denote the orientation of the magnetic moments in the subsurface Gd layer.

$\overline{\mathrm{M}}-\bar{\Gamma}$ is related to $\overline{\mathrm{M}}-\overline{\mathrm{X}}$ by an anticlockwise rotation around $\bar{M}$ by $\pi / 4$, whereby the cubic Rashba effect (triple winding) of the $\alpha$ state is seen by the change of the sign of the $S_{y}$ component: along $\overline{\mathrm{M}}-\bar{\Gamma}$, the inner branch becomes red and the outer becomes blue [Fig. 5(a)] indicating the rotation of the spin by $3 \pi / 4$ (see Fig. 4 as well).

Let us now discuss the surface state modifications upon switching on the exchange field, that is going to compete with the Rashba SOC field. Like for the Rashba SOC the exchange interaction can be described by an effective magnetic field, the exchange field $\mathbf{B}_{\mathrm{ex}}$, which is parallel to the magnetization. The final direction of the spins is going to be defined by the total magnetic field $\mathbf{B}_{\text {tot }}=\mathcal{B}_{\mathrm{R}}+\mathbf{B}_{\text {ex }}$, which is $k$-dependent. The resulting spin texture and band dispersion will thus depend on the mutual alignment and relative strength of the two fields.
For the AFM phase we consider first the [100] magnetization direction, Fig. 5(b). The CECs are shown for an energy of $-0.15 \mathrm{eV}$. From the spin polarization of the PM phase we know, that for the $\overline{\mathrm{X}}-\overline{\mathrm{M}}-\overline{\mathrm{X}} \| k_{x}$ path, 1 , the Rashba field is collinear with the [010] direction [Fig. 5(a-1)]. The exchange field, in turn, tends to align the spins with the [100] direction. (At this point is important to keep in mind, that the spin and the magnetic moment of the spin are antiparallel. Thus, it is the spin magnetic moment which favors a parallel alignment with respect to the exchange field, i.e., along the [100] direction, while the spin favors an antiparallel orientation.) As a result, the spins get tilted by the exchange field and acquire a large $S_{x}$ component at the expense of $S_{y}$, see Fig. 5(b-1). Meanwhile, the $S_{y}$ spin component qualitatively preserves its pattern characteristic for the cubic Rashba effect and not only for this direction but for all $\mathbf{k}$, cf. spin-resolved CECs 
in Figs. 5(a) and 5(b). Since for the $\bar{X}-\bar{M}-\bar{X} \| k_{x}$ path the exchange and Rashba fields are orthogonal, the splitting of the surface state is symmetric with respect to the $\bar{M}$ point (like in the PM case), and the emerging $S_{x}$ polarization is symmetric as well. Moreover, the exchange interaction increases the overall splitting of the surface state bands as compared to the PM phase. Note that for the $S_{x}$ spin component for both (left and right) branches of the $\alpha$ state the lower band is build by $+S_{x}$ while the upper band by $-S_{x}$ [Fig. 5(b-1)]. This is consistent with the picture seen for the scalar relativistic AFM case, where the $+S_{x}\left(-S_{x}\right)$ spins represent the majority (minority) channel (see Ref. [39], Fig. S7).

For the $\bar{\Gamma}-\overline{\mathrm{M}}-\bar{\Gamma}$ direction, the Rashba and exchange fields form angles of $\pm 45^{\circ}$, see Figs. 4(c) and 5(b-2), whereby the $\alpha$ state demonstrates nonzero $S_{x}$ and $S_{y}$ polarization simultaneously. On the left from the $\overline{\mathrm{M}}$ point, upon switching on the exchange field the Rashba-split bands carrying $+S_{x}\left(-S_{x}\right)$ spin components experience a notable downward (upward) shift. This displaces the outer (inner) contour's line away from (towards to) the $\overline{\mathrm{M}}$ point. However, on the right of the $\overline{\mathrm{M}}$ point the splitting appears to be reduced, because the directions of the Rashba field and, therefore, the directions of the spins for these bands in the PM state are opposite to those on the left of $\overline{\mathrm{M}}$. Consequently, the contours come closer one to another as a result of the application of the exchange field. This creates a pronounced asymmetry with respect to the $\overline{\mathrm{M}}$ point along the $\bar{\Gamma}-\bar{M}-\bar{\Gamma}$ direction. Besides, the spins of both bands on the right of $\bar{M}$ experience a clockwise rotation upon the exchange field action due to which the $S_{x}$ components of the bands get reversed (as compared to their signs in the pure Rashba effect), while the $S_{y}$ components maintain their order, but grow in absolute value. Note, that a similar argumentation applies to the other $\bar{\Gamma}-\overline{\mathrm{M}}-\bar{\Gamma}$ path [Fig. 5(b-4)].

For the $\overline{\mathrm{X}}-\overline{\mathrm{M}}-\overline{\mathrm{X}} \| k_{y}$ path presented in Fig. 5(a-3) the alignment of the spins in the PM phase is already collinear with the emerging exchange field. Therefore, the spin polarization of the PM phase remains unaltered in Fig. 5(b-3) but the spin-polarized states are shifted in energy by the exchange field: states with spin antiparallel to the exchange field $\left(S_{x}>0\right)$ are lowered, while states with their spin parallel $\left(S_{x}<0\right)$ are raised. Since the bands on the left and right of the $\bar{M}$ point have opposite spin polarization, this results in the most pronounced asymmetry in the dispersion of the $\alpha$ state. Namely, for its left lower branch featuring $S_{x}<0$ due to the Rashba effect, the energy is raised, while for the left upper branch characterized by $S_{x}>0$ the energy is lowered, which results in a crossing of the bands. For the right branch the opposite situation is observed and the splitting is strongly enhanced.

Overall, the results shown in Fig. 5(b) can be summarized as follows. Application of the exchange field along the [100] direction causes the spins on the outer (inner) contour of the Rashba-split $\alpha$ state to rotate towards [100] ([100]). In the limit of a negligibly weak SOC, the spins on the outer and inner contours would be perfectly aligned with [100] and [100] directions, respectively (see Ref. [39], Fig. S7). However, the strong SOC of the Ir- $d$ states, which significantly contribute to the $\alpha$ band, counteracts the exchange field, leading to the formation of the peculiar spin structure shown in Fig. 6(a). At that, the two contours can be considered as independent in the following sense. First, there are no crossings between them (although they come close to one another slightly off $\bar{\Gamma}-\bar{M}-\bar{\Gamma}$ ) and, second, the rotations of the spins can be smoothly followed within each contour separately. Overall, as shown in Fig. 6(b), the direction of deformations of the contours depends on how the Rashba field aligns the spin relative to the emerging exchange field. Namely, the segments of the contours that feature spins pointing parallel (antiparallel) to the exchange field shrink (expand). The description that we have given here for the $\alpha$-state's CEC at $-0.15 \mathrm{eV}$ holds for any other of its CECs down to an energy of about $-0.38 \mathrm{eV}$, at which the crossings of the otherwise spinsplit bands occur along the $\overline{\mathrm{X}}-\overline{\mathrm{M}}-\overline{\mathrm{X}} \| k_{y}$ direction [Fig. 5(b-3)] as well as slightly off the $\bar{\Gamma}-\bar{M}-\bar{\Gamma}$ direction (see Ref. [39], Fig. S8). In this case, one cannot define two separate contours and, at the crossing points, the spin texture shows a smooth behavior only when changing from the outer line to the inner (i.e., always "staying" at either outer or inner line only when going along the CEC disrupts the smooth spin rotation upon passing the crossings).

Let us now discuss the results obtained for the [ $\overline{11} 0]$ direction of the exchange field. While the Rashba field is unaltered, the exchange field is now rotated by $45^{\circ}$ as compared to the [100] magnetization case considered above. Therefore it acts equally on both in-plane spin components $S_{x}$ and $S_{y}$. Figure 5(c) shows typical CECs for this case, which clearly shows that the contours are not independent, but they feature three crossings. Interestingly, for this exchange field direction, there is no such energy at which two clearly distinct contours, inner and outer, could be distinguished: there are always touching points present.

In the PM phase, the $\alpha$ state features only the $S_{y}$ spin component along the $\overline{\mathrm{X}}-\overline{\mathrm{M}}-\overline{\mathrm{X}} \| k_{x}$ direction. The exchange field emerging along [110] acts on the $\pm S_{y}$ spins with its $y$ component (which is negative), shifting downwards (upwards) in energy the $+S_{y}\left(-S_{y}\right)$ states [Figs. 5(c-1) and 6(c)]. This creates a significant asymmetry in the CECs. Besides, the spins get rotated towards the [110] $([\overline{110}])$ direction for the outer (inner) branch, and a nonzero $S_{x}$ component emerges. Similar analysis is applicable to the $\overline{\mathrm{X}}-\overline{\mathrm{M}}-\overline{\mathrm{X}} \| k_{y}$ direction [Figs. 5(c-3) and 6(c)].

For the $\bar{\Gamma}-\overline{\mathrm{M}}-\bar{\Gamma}$ path, which is parallel to the magnetization direction the exchange and Rashba fields are orthogonal. As a result, a symmetric band dispersion can be seen, with the spin splitting slightly enhanced as compared to the PM phase [Fig. 5(c-2)]. The spins of the outer (inner) branches rotate towards the direction antiparallel (parallel) to that of the exchange field [Fig. 6(c)].

A pronounced asymmetry is also seen for the $\bar{\Gamma}-\overline{\mathrm{M}}-\bar{\Gamma}$ path, which is orthogonal to the magnetization direction [Fig. 5(c-4)]. For this case, the Rashba and exchange fields are collinear, which leads to the contours moving away from one another on the left of $\overline{\mathrm{M}}$, but coming together and forming two crossing points on its right. Thus, despite the fact that for the [110] magnetization direction the changes in the dispersion and spin structure of the $\alpha$ state are more complicated, they also can be explained in terms of the exchange field's tendency to lower (increase) the energy of the states 

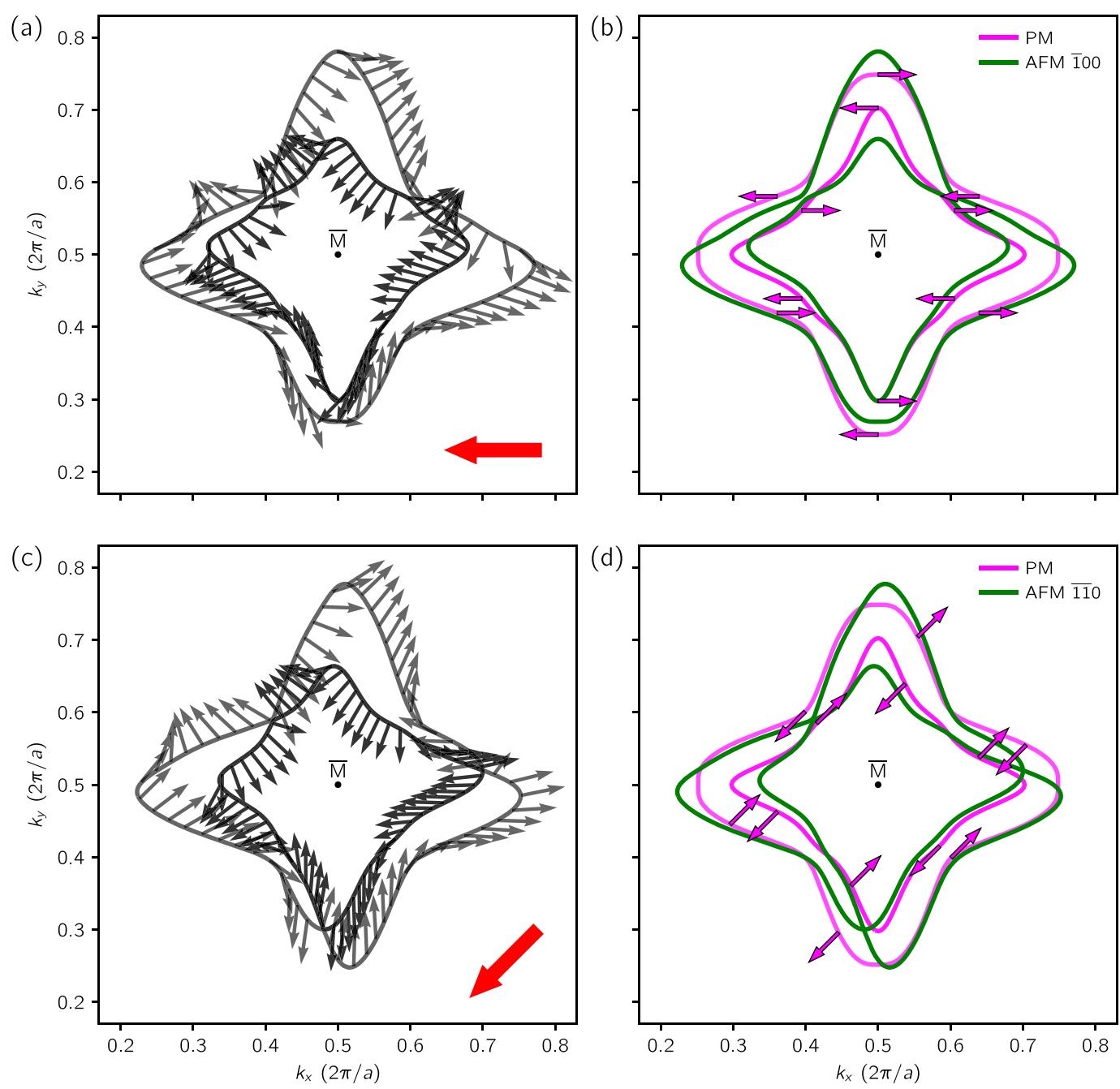

FIG. 6. The spin-resolved CECs for the $\alpha$ state calculated at $-0.15 \mathrm{eV}$ for (a) the $[\overline{1} 00]$ and (c) the [110] magnetization direction. The red arrows indicate the orientation of the ordered $4 f$ moments in the fourth layer. [(b) and (d)] Schematic illustration of the deformation of the Rashba-split CECs (magenta) upon application of the exchange field (green). The magenta-colored arrows show the points on the contour where the spins are collinear to the emergent exchange field and where, therefore, strong distortions of the CECs can be generally expected.

with spin antiparallel (parallel) to the magnetization direction, Fig. 6(d).

\section{Linear Rashba effect and in-plane exchange field ( $\gamma$ state)}

All general conclusions from the analysis of the mutual orientation of the exchange and Rashba fields for the $\alpha$ state are valid for the $\gamma$ state as well. So, we concentrate on the discussion of differences between the $\gamma$ and $\alpha$ states. In the PM phase, the $\gamma$ state has the spin structure characteristic for the linear Rashba effect, as can be seen from Fig. 5(d). For $\overline{\mathrm{X}}-\overline{\mathrm{M}}-\overline{\mathrm{X}}$, the spin polarization of $\gamma$ and $\alpha$ has opposite sign, while the sign is the same for $\bar{\Gamma}-\overline{\mathrm{M}}-\bar{\Gamma}$, reflecting the fundamental difference between a linear and a cubic Rashba system.

In the AFM phase for both magnetization directions, the spin splitting of the $\gamma$ state experiences weaker changes than those of the $\alpha$ state, and the rotation of the spins in direction of the exchange field is weaker. The latter can be seen from comparison of the spin structure for the PM and AFM cases as well as from the analysis of the spin components emerging under the exchange field action, which align along the magnetization direction. It can be explained by the fact that the $\gamma$ state is located within the two topmost layers ( $\mathrm{Si}$ and Ir) and has thus a negligible overlap with the Gd atomic layer, whereby the interaction of the $\gamma$ state with the ordered $4 f$ moments differs significantly from the case of the surface state $\alpha$. Such a localization of the $\gamma$ state can mean that it couples indirectly to the ordered $4 f$ moments. Interestingly, for $\gamma$ the exchange coupling is strongly energy dependent and even changes sign at an energy $E=-50 \mathrm{meV}$. For this energy, the exchange coupling vanishes and only the effective Rashba field acts on the electrons of the $\gamma$ state. Hence, no asymmetric distortion of the CECs occurs. Asymmetries can only evolve for a nonzero exchange coupling, that is, only at energies lower or higher than $-50 \mathrm{meV}$, and the strength of the asymmetric distortion is energy dependent, too. For a detailed discussion we refer to Ref. [39], Fig. S7 and related discussion. 

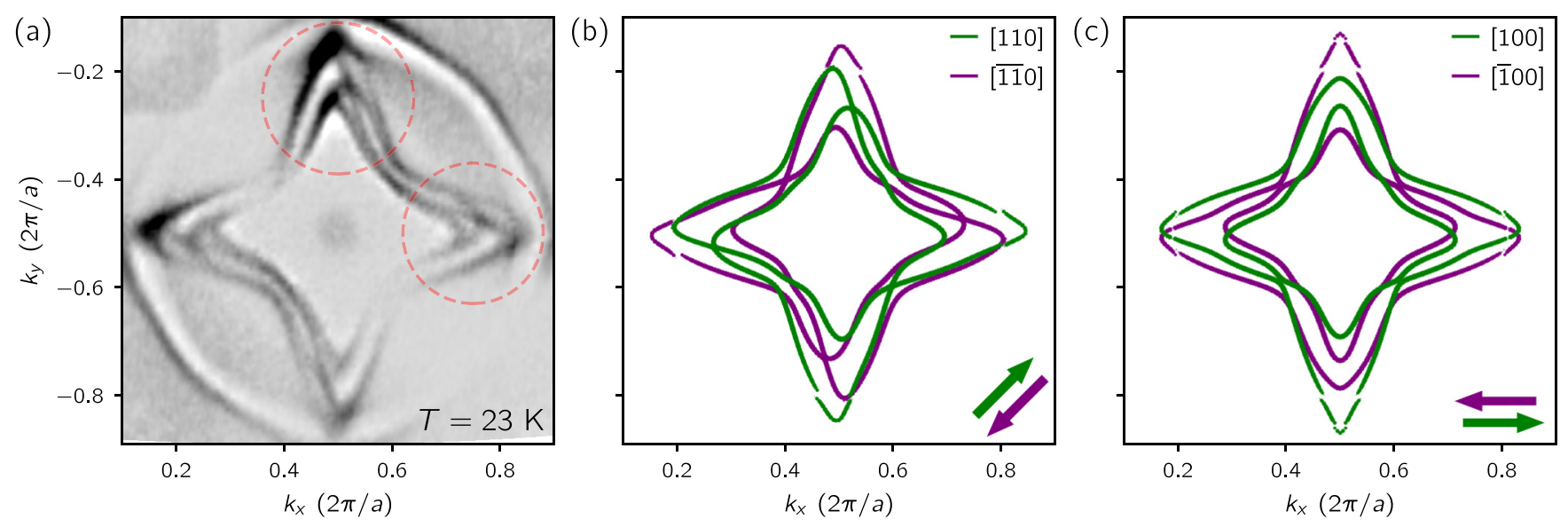

FIG. 7. (a) ARPES-derived Fermi map of Fig. 3(d) and (b) DFT results for the $\alpha$ state's Fermi surface for the case of antiparallel magnetic domains with magnetization along the $[\overline{110}]$ and [110] directions, shown in purple and green, respectively. (c) $\alpha$ state for magnetization along [100] (green) and [100] (purple). Comparison of $\alpha$ as it is seen in ARPES and DFT shows the best agreement for magnetization along [110] given in (b). This can be particularly well seen for the corners of the surface state $\alpha$ that are marked by red-dashed circles in the ARPES map in (a).

Since the modifications of the spin polarization under the action of the exchange field are small if compared to the $\alpha$ state, we may assume that the exchange coupling is notably weaker for $\gamma$. There, only in the vicinity of the $\bar{M}$ point, where the Rashba field vanishes, a pronounced change in the spin polarization occurs in $\overline{\mathrm{X}}-\overline{\mathrm{M}}-\overline{\mathrm{X}}$ direction. Comparison of Figs. 5(d-1) with Figs. 5(e-1) and 5(f-1) shows that close to $\bar{M}$ the spins acquire a notable $S_{x}$ component. For magnetization along [110], an $S_{y}$ polarization emerges accordingly [compare panel 3 of Figs. 5(f) and 5(d)].

\section{Multiple domains and direction of magnetization}

Having so far discussed in detail the spin structure and asymmetry caused by the combined action of SOC and exchange interaction, we will now come back to the question of the direction of the magnetization as well as the problem of having multiple domains.

As we already mentioned, the stepped surface of the freshly cleaved $\mathrm{GdIr}_{2} \mathrm{Si}_{2}$ complicates the measurement of a single magnetic domain in a conventional ARPES experiment due to the antiferromagnetic ordering of the Gd layers. Hence, in the simplest case, one may expect that the photoemission signal stems from the superposition of two antiparallel magnetic domains. Based on this assumption, we show in Figs. 7(b) and 7 (c) the resulting CECs of the $\alpha$ state as seen for two opposite domains of the [110] and [100] directions. The main and most obvious difference between the two directions are the diagonal and vertical symmetry axes seen for [110] and [100], respectively, which are preserved in the case of antiparallel domains. Hence, the differences are most pronounced at the corners of the star-like shaped CECs. The opening of the small gaps close to the tips of the outermost CECs is the result of the hybridization with the surface-resonant bulk bands.

Comparing the contours of both directions to our measured Fermi map shown in Fig. 7(a), we can again carefully look at the corners of surface state $\alpha$, highlighted in red. There, the asymmetric behavior and the clearly visible band crossings show strong similarities to the computational results for the
[110] direction, Fig. 7(b). Thus, our measurements suggest that at the given temperature of about $23 \mathrm{~K}$, the direction of magnetization in $\mathrm{GdIr}_{2} \mathrm{Si}_{2}$ is along [110] and not along [100]. Further, the absence of contributions from perpendicular domains with magnetization along [ $\overline{1} 10]$ and $[1 \overline{1} 0]$ indicates that the magnetic domains in the bulk are larger than the spot size of the probing photon beam and supports our assumption that the band doublings are primarily caused by the coupling of the stepped surface to the underlying AFM order.

However, in light of $\mathrm{GdRh}_{2} \mathrm{Si}_{2}$ with its temperaturedependent magnetization direction [32], we can neither exclude here that the magnetization direction changes temperature-dependently nor that the magnetization direction is slightly off the high-symmetry direction [110]. Nevertheless, our results instructively show that in such an intricate case with multiple domains, the surface state and its asymmetries caused by exchange interaction and SOC still allow to infer information about the direction of the magnetization.

\section{CONCLUSION}

Extensive and long-term studies of $\mathrm{RET}_{2} \mathrm{Si}_{2}$ compounds showed, that the surface Si-T-Si-RE block forms a promising system for studying the Rashba-like spin-orbit coupling and its joint action with exchange magnetism on two-dimensional charge carriers. Particularly, the surface states located in a large projected band gap around the $\overline{\mathrm{M}}$ point attracted great interest. To combine a strong spin-orbit coupling with exchange magnetic interaction it seems logical to bring together heavy Ir with magnetically active $\mathrm{Gd}$ within the respective $\mathrm{Si}-\mathrm{T}-\mathrm{Si}-\mathrm{RE}$ block. Due to its half-filled $4 f$ shell, implying $L=0$, and therefore pure spin moment $J=S=7 / 2, \mathrm{Gd}$ is the simplest magnetically active rare-earth element which is rather insensitive to the crystal-electric-field environment. Following this chain of thought, we arrived at the system $\mathrm{GdIr}_{2} \mathrm{Si}_{2}$, which is a layered antiferromagnetic material. We thus performed a study of the surface electronic structure of $\mathrm{GdIr}_{2} \mathrm{Si}_{2}$ in the PM and AFM phases, thereby focusing on the surface states $\alpha$ and $\gamma$ located in the large band gap around the $\overline{\mathrm{M}}$ point of 
the SBZ. In accordance with our expectations and with former studies on the valence-fluctuating system $\mathrm{EuIr}_{2} \mathrm{Si}_{2}$, we find the surface state electrons to be subject to a strong Rashba effect, which leads to highly spin-polarized and strongly spin-split bands. Here, only the $\gamma$ state shows the spin structure of a classical, linear Rashba effect. In turn, the $\alpha$ band shows a so-called cubic Rashba effect, which is characterized by a triple winding of the spin around the CECs. The origin of the different spin character lies in the orbital composition of the surface states. Further, we found that with magnetic ordering of the $4 f$ moments the spin structure gets strongly modified and asymmetries occur in the band dispersion and thus, also in the CECs. Both the Rashba-like spin-orbit interaction and the exchange interaction can be described by effective magnetic fields, a $k$ dependent Rashba field and the exchange field. Considering the resulting total field allows understanding the complex spin structures of the aforementioned surface states in the PM and AFM phases. The wave function of the $\alpha$ state extends over the whole Si-Ir-Si-Gd surface block. The overlap with the Gd layer results in a direct exchange coupling of the itinerant spins to the localized $4 f$ moments. Since the $\gamma$ state concentrates within the topmost $\mathrm{Si}$-Ir two-layer it has a negligible overlap with $\mathrm{Gd}$, suggesting an indirect coupling to the ordered $4 f$ moments, which is strongly energy-dependent.

We have also shown and discussed that the formation of a mosaic of magnetic domains essentially complicates the analysis of ARPES patters in comparison to the single-domain case observed for $\mathrm{EuIr}_{2} \mathrm{Si}_{2}$ [6] but as we show here it is still possible to derive the orientation of the easy-magnetization axis even for the multi-domain case. It is assumed that further insight into the properties of a single domain can be obtained by means of an alternative experimental technique, namely with nano-ARPES. The formation of magnetic domains and domain walls might also be of interest for further investigations, e.g. in the frame of spin-resolved PEEM experiments.
Another point is that similar to the $\mathrm{GdRh}_{2} \mathrm{Si}_{2}$ system, for $\mathrm{GdIr}_{2} \mathrm{Si}_{2}$ may also exist the temperature window where the surface and bulk magnetic systems experience only weak coupling to each other [12]. If this is the case, the application of a relatively small external magnetic field could help to re-orient the magnetic domains within the surface, which would simplify spectroscopic measurements aiming at the investigation of the magnetic properties of the surface and the bulk system of this compound.

\section{ACKNOWLEDGMENTS}

This work was supported by the German Research Foundation (DFG) through Grants No. KR3831/5-1, No. LA655/201, No. GRK1621, No. SFB1143 (Project No. 247310070). We acknowledge support from the Russian Foundation for Basic Research (Grant No. 20-32-70127) and Saint Petersburg State University (Grant No. ID 51126254). M.M.O. acknowledges the support by Spanish Ministerio de Ciencia e Innovación (Grant No. PID2019-103910GB-I00). A.Yu.V. acknowledges the support by RFBR, Project No. 19-32-90251. D.V.V. acknowledges financial support from the Spanish Ministry of Economy (MAT-2017-88374-P). We acknowledge MAX IV Laboratory for time on the Bloch Beamline under Proposal 20190824. Research conducted at MAX IV, a Swedish national user facility, is supported by the Swedish Research council under contract 2018-07152, the Swedish Governmental Agency for Innovation Systems under contract 2018-04969, and Formas under contract 2019-02496. The authors gratefully acknowledge the GWK support for funding this work by providing computing time through the Center for Information Services and HPC (ZIH) at TU Dresden. Additional calculations were performed in the Research park of St. Petersburg State University Computing Center (http: //cc.spbu.ru).
[1] Yu. A. Bychkov and E. I. Rashba, Properties of a 2D electron gas with lifted spectral degeneracy, ZhETF Pisma Redaktsiiu 39, 66 (1984) [JETP Lett 39, 78 (1984)].

[2] E. I. Rashba, Properties of semiconductors with an extremum loop. I. Cyclotron and combinational resonance in a magnetic field perpendicular to the plane of the loop, Sov. Phys. Solid State 2, 1109 (1960).

[3] Yu. A. Bychkov and E. I. Rashba, Oscillatory effects and the magnetic susceptibility of carriers in inversion layers, J. Phys. C 17, 6039 (1984).

[4] S. LaShell, B. A. McDougall, and E. Jensen, Spin Splitting of an Au (111) Surface State Band Observed with Angle Resolved Photoelectron Spectroscopy, Phys. Rev. Lett. 77, 3419 (1996).

[5] A. Generalov, J. Falke, I. A. Nechaev, M. M. Otrokov, M. Güttler, A. Chikina, K. Kliemt, S. Seiro, K. Kummer, S. Danzenbächer, D. Usachov, T. K. Kim, P. Dudin, E. V. Chulkov, C. Laubschat, C. Geibel, C. Krellner, and D. V. Vyalikh, Strong spin-orbit coupling in the noncentrosymmetric Kondo lattice, Phys. Rev. B 98, 115157 (2018).

[6] S. Schulz, I. A. Nechaev, M. Güttler, G. Poelchen, A. Generalov, S. Danzenbächer, A. Chikina, S. Seiro, K. Kliemt, A. Yu. Vyazovskaya, T. K. Kim, P. Dudin, E. V. Chulkov,
C. Laubschat, E. E. Krasovskii, C. Geibel, C. Krellner, K. Kummer, and D. V. Vyalikh, Emerging 2D-ferromagnetism and strong spin-orbit coupling at the surface of valence-fluctuating EuIr $\mathrm{Si}_{2}$, npj Quantum Mater. 4, 26 (2019).

[7] D. Yu. Usachov, I. A. Nechaev, G. Poelchen, M. Güttler, E. E. Krasovskii, S. Schulz, A. Generalov, K. Kliemt, A. Kraiker, C. Krellner, K. Kummer, S. Danzenbächer, C. Laubschat, A. P. Weber, J. Sánchez-Barriga, E. V. Chulkov, A. F. SantanderSyro, T. Imai, K. Miyamoto, T. Okuda, and D. V. Vyalikh, Cubic Rashba Effect in the Surface Spin Structure of Rare-Earth Ternary Materials, Phys. Rev. Lett. 124, 237202 (2020).

[8] W. Lin, L. Li, F. Doğan, C. Li, H. Rotella, X. Yu, B. Zhang, Y. Li, W. S. Lew, S. Wang, W. Prellier, S. J. Pennycook, J. Chen, Z. Zhong, A. Manchon, and T. Wu, Interface-based tuning of Rashba spin-orbit interaction in asymmetric oxide heterostructures with $3 d$ electrons, Nat. Commun. 10, 3052 (2019).

[9] M. Höppner, S. Seiro, A. Chikina, A. Fedorov, M. Güttler, S. Danzenbächer, A. Generalov, K. Kummer, S. Patil, S. L. Molodtsov, Yu. Kucherenko, C. Geibel, V. N. Strocov, M. Shi, M. Radovic, T. Schmitt, C. Laubschat, and D. V. Vyalikh, Interplay of Dirac fermions and heavy quasiparticles in solids, Nat. Commun. 4, 1646 (2013). 
[10] M. Güttler, K. Kummer, S. Patil, M. Höppner, A. Hannaske, S. Danzenbächer, M. Shi, M. Radovic, E. Rienks, C. Laubschat, C. Geibel, and D. V. Vyalikh, Tracing the localization of $4 f$ electrons: Angle-resolved photoemission on $\mathrm{YbCo}_{2} \mathrm{Si}_{2}$, the stable trivalent counterpart of the heavy-fermion $\mathrm{YbRh}_{2} \mathrm{Si}_{2}$, Phys. Rev. B 90, 195138 (2014).

[11] A. Chikina, M. Höppner, S. Seiro, K. Kummer, S. Danzenbächer, S. Patil, A. Generalov, M. Güttler, Y. Kucherenko, E. V. Chulkov, Y. M. Koroteev, K. Koepernik, C. Geibel, M. Shi, M. Radovic, C. Laubschat, and D. V. Vyalikh, Strong ferromagnetism at the surface of an antiferromagnet caused by buried magnetic moments, Nat. Commun. 5, 3171 (2014).

[12] M. Güttler, A. Generalov, M. M. Otrokov, K. Kummer, K. Kliemt, A. Fedorov, A. Chikina, S. Danzenbächer, S. Schulz, E. V. Chulkov, Y. M. Koroteev, N. Caroca-Canales, M. Shi, M. Radovic, C. Geibel, C. Laubschat, P. Dudin, T. K. Kim, M. Hoesch, C. Krellner, and D. V. Vyalikh, Robust and tunable itinerant ferromagnetism at the silicon surface of the antiferromagnet $\mathrm{GdRh}_{2} \mathrm{Si}_{2}$, Sci. Rep. 6, 24254 (2016).

[13] A. Chikina, A. Generalov, K. Kummer, M. Güttler, V. N. Antonov, Yu. Kucherenko, K. Kliemt, C. Krellner, S. Danzenbächer, T. Kim, P. Dudin, C. Geibel, C. Laubschat, and D. V. Vyalikh, Valence instability in the bulk and at the surface of the antiferromagnet $\mathrm{SmRh}_{2} \mathrm{Si}_{2}$, Phys. Rev. B 95, 155127 (2017).

[14] A. Generalov, M. M. Otrokov, A. Chikina, K. Kliemt, K. Kummer, M. Höppner, M. Güttler, S. Seiro, A. Fedorov, S. Schulz, S. Danzenbächer, E. V. Chulkov, C. Geibel, C. Laubschat, P. Dudin, M. Hoesch, T. Kim, M. Radovic, M. Shi, N. C. Plumb, C. Krellner, and D. V. Vyalikh, Spin orientation of two-dimensional electrons driven by temperature-tunable competition of spin-orbit and exchange-magnetic interactions, Nano Lett. 17, 811 (2017).

[15] A. Yu. Vyazovskaya, M. M. Otrokov, Yu. M. Koroteev, K. Kummer, M. Güttler, D. V. Vyalikh, and E. V. Chulkov, Origin of two-dimensional electronic states at Si-and Gd-terminated surfaces of $\mathrm{GdRh}_{2} \mathrm{Si}_{2}$ (001), Phys. Rev. B 100, 075140 (2019).

[16] I. A. Nechaev and E. E. Krasovskii, Relativistic splitting of surface states at $\mathrm{Si}$-terminated surfaces of the layered intermetallic compounds $R T_{2} \mathrm{Si}_{2}$ ( $R=$ rare earth; $T=\mathrm{Ir}, \mathrm{Rh}$ ), Phys. Rev. B 98, 245415 (2018).

[17] M. Shatruk, $\mathrm{ThCr}_{2} \mathrm{Si}_{2}$ structure type: The "perovskite" of intermetallics, J. Solid State Chem. 272, 198 (2019).

[18] D. Yu. Usachov, A. V. Tarasov, S. Schulz, K. A. Bokai, I. I. Tupitsyn, G. Poelchen, S. Seiro, N. Caroca-Canales, K. Kliemt, M. Mende, K. Kummer, C. Krellner, M. Muntwiler, H. Li, C. Laubschat, C. Geibel, E. V. Chulkov, S. I. Fujimori, and D. V. Vyalikh, Photoelectron diffraction for probing valency and magnetism of $4 f$-based materials: A view on valencefluctuating EuIr $\mathrm{Si}_{2}$, Phys. Rev. B 102, 205102 (2020).

[19] L. Wang and M. W. Wu, Hole spin relaxation in p-type (111) GaAs quantum wells, Phys. Rev. B 85, 235308 (2012).

[20] P. Höpfner, J. Schäfer, A. Fleszar, J. H. Dil, B. Slomski, F. Meier, C. Loho, C. Blumenstein, L. Patthey, W. Hanke, and R. Claessen, Three-Dimensional Spin Rotations at the Fermi Surface of a Strongly Spin-Orbit Coupled Surface System, Phys. Rev. Lett. 108, 186801 (2012).

[21] K. Kliemt, M. Peters, F. Feldmann, A. Kraiker, D.-M. Tran, S. Rongstock, J. Hellwig, S. Witt, M. Bolte, and C. Krellner,
Crystal growth of materials with the $\mathrm{ThCr}_{2} \mathrm{Si}_{2}$ structure type, Cryst. Res. Technol. 55, 1900116 (2020).

[22] D. Gignoux and D. Schmitt, Magnetism of compounds of rare earths with non-magnetic metals, Handbook of magnetic materials 10, 239 (1997).

[23] A. Szytuła and J. Leciejewicz, Magnetic properties of ternary intermetallic compounds of the $\mathrm{RT}_{2} \mathrm{X}_{2}$ type, Handbook on the Physics and Chemistry of Rare Earths 12, 133 (1989).

[24] O. Krupin, G. Bihlmayer, K. Starke, S. Gorovikov, J. E. Prieto, K. Döbrich, S. Blügel, and G. Kaindl, Rashba effect at magnetic metal surfaces, Phys. Rev. B 71, 201403(R) (2005).

[25] O. Krupin, G. Bihlmayer, K. Döbrich, J. Prieto, K. Starke, S. Gorovikov, S. Blügel, S. Kevan, and G. Kaindl, Rashba effect at the surfaces of rare-earth metals and their monoxides, New J. Phys. 11, 013035 (2009).

[26] I. I. Klimovskikh, A. M. Shikin, M. M. Otrokov, A. Ernst, I. P. Rusinov, O. E. Tereshchenko, V. A. Golyashov, J. SánchezBarriga, A. Y. Varykhalov, O. Rader, K. A. Kokh, and E. V. Chulkov, Giant magnetic band gap in the Rashba-split surface state of vanadium-doped BiTeI: A combined photoemission and ab initio study, Sci. Rep. 7, 3353 (2017).

[27] A. G. Rybkin, A. A. Rybkina, M. M. Otrokov, O. Y. Vilkov, I. I. Klimovskikh, A. E. Petukhov, M. V. Filianina, V. Y. Voroshnin, I. P. Rusinov, A. Ernst, A. Arnau, E. V. Chulkov, and A. M. Shikin, Magneto-spin-orbit graphene: interplay between exchange and spin-orbit couplings, Nano Lett. 18, 1564 (2018).

[28] D. Yu. Usachov, M. Güttler, S. Schulz, G. Poelchen, S. Seiro, K. Kliemt, K. Kummer, C. Krellner, C. Laubschat, E. V. Chulkov, and D. V. Vyalikh, Spin structure of spin-orbit split surface states in a magnetic material revealed by spin-integrated photoemission, Phys. Rev. B 101, 245140 (2020).

[29] B. Chevalier, J. M. D. Coey, B. Lloret, and J. Etourneau, $\mathrm{EuIr}_{2} \mathrm{Si}_{2}$ : A new intermediate valence compound, J. Phys. C 19, 4521 (1986).

[30] S. Seiro and C. Geibel, From stable divalent to valencefluctuating behavior in $\mathrm{Eu}\left(\mathrm{Rh}_{1-\mathrm{x}} \mathrm{Ir}_{\mathrm{x}}\right)_{2} \mathrm{Si}_{2}$ single crystals, J. Phys. Condens. Matter 23, 375601 (2011).

[31] T. T. Jungwirth, J. Sinova, A. Manchon, X. Marti, J. Wunderlich, and C. Felser, The multiple directions of antiferromagnetic spintronics, Nat. Phys. 14, 200 (2018).

[32] Y. W. Windsor, A. Ernst, K. Kummer, K. Kliemt, C. SchüßlerLangeheine, U. Pontius, U. Staub, E. V. Chulkov, C. Krellner, D. V. Vyalikh, and L. Rettig, Deterministic control of an antiferromagnetic spin arrangement using ultrafast optical excitation, Comm. Physics 3, 139 (2020).

[33] B. Böhm, L. Fallarino, D. Pohl, B. Rellinghaus, K. Nielsch, N. S. Kiselev, and O. Hellwig, Antiferromagnetic domain wall control via surface spin flop in fully tunable synthetic antiferromagnets with perpendicular magnetic anisotropy, Phys. Rev. B 100, 140411(R) (2019).

[34] T. Shiino, S.-H. Oh, P. M. Haney, S.-W. Lee, G. Go, B.-G. Park, and K.-J. Lee, Antiferromagnetic Domain Wall Motion Driven by Spin-Orbit Torques, Phys. Rev. Lett. 117, 087203 (2016).

[35] Bloch beamline, MAX IV, https://www.maxiv.lu.se/ accelerators-beamlines/beamlines/bloch/.

[36] K. Koepernik and H. Eschrig, Full-potential nonorthogonal local-orbital minimum-basis band-structure scheme, Phys. Rev. B 59, 1743 (1999). 
[37] G. Kresse and J. Furthmüller, Efficient iterative schemes for ab initio total-energy calculations using a plane-wave basis set, Phys. Rev. B 54, 11169 (1996).

[38] G. Kresse and D. Joubert, From ultrasoft pseudopotentials to the projector augmented-wave method, Phys. Rev. B 59, 1758 (1999).

[39] See Supplemental Material at http://link.aps.org/supplemental/ 10.1103/PhysRevB.103.035123 for ARPES spectra of the Gdterminated surface, the influence of the interlayer spacing between the topmost $\mathrm{Si}$ and adjacent Ir layer on the calculated energy position of the surface state bands, a visualization of the two crystallographic nonequivalent Ir sites, the dispersion of the surface state bands if SOC and magnetic order are neglected, the relation between spin structure and orbital composition of the surface states, the dispersion and spin structure of the surface state bands for a scalar-relativistic computational setting with AFM order and the calculated spin-resolved CECs for the energy $-0.38 \mathrm{eV}$.

[40] P. Zhang, P. Richard, T. Qian, Y.-M. Xu, X. Dai, and H. Ding, A precise method for visualizing dispersive features in image plots, Rev. Sci. Instrum. 82, 043712 (2011).

[41] X. Zhang, Q. Liu, J. W. Luo, A. J. Freeman, and A. Zunger, Hidden spin polarization in inversion-symmetric bulk crystals, Nat. Phys. 10, 387 (2014).

[42] I. A. Nechaev and E. E. Krasovskii, Ab initio k $\cdot \mathbf{p}$ theory of spin-momentum locking: Application to topological surface states, Phys. Rev. B 102, 115437 (2020). 